\title{
Stability and superconductivity of lanthanum and yttrium decahydrides
}

\author{
Alice M. Shipley, ${ }^{*}$ Michael J. Hutcheon $\dagger^{\dagger}$ Mark S. Johnson, and Richard J. Needs \\ Theory of Condensed Matter Group, Cavendish Laboratory, \\ J. J. Thomson Avenue, Cambridge CB3 OHE, United Kingdom
}

\author{
Chris J. Pickard \\ Department of Materials Science and Metallurgy, \\ 27 Charles Babbage Rd, Cambridge CB3 OFS, United Kingdom and \\ Advanced Institute for Materials Research, Tohoku University, \\ 2-1-1 Katahira, Aoba, Sendai, 980-8577, Japan
}

\begin{abstract}
Rare-earth hydrides can exhibit high-temperature superconductivity under high pressure. Here, we apply a crystal structure prediction method to the current record-holding $T_{c}$ material, $\mathrm{LaH}_{10}$. We find a pressure-induced phase transition from the experimentally observed cubic phase to a hexagonal phase at around $420 \mathrm{GPa}$. This new phase is metastable down to low pressures and could explain experimental observations of hcp impurities in fcc samples. We go on to find that $\mathrm{YH}_{10}$ adopts similar structures and discuss the sensitivity of superconductivity calculations to certain computational parameters.
\end{abstract}

\section{INTRODUCTION}

Hydrogen was predicted to be a room-temperature superconductor at very high pressure in 1968 [1, but the pressures required to metallise hydrogen are difficult to obtain [2 7]. Hydrides have been suggested to have lower metallisation pressures than pure hydrogen due to chemical pre-compression [8] and therefore might become superconducting at more readily accessible pressures. This idea has motivated a surge of research examining potential superconductivity in high-pressure hydrides, with several reviews summarising recent developments [9] 15 .

Theoretical studies of $\mathrm{ScH}_{3}, \mathrm{LaH}_{3}$ [16, $\mathrm{YH}_{3}, \mathrm{YH}_{4}$ and $\mathrm{YH}_{6}$ [17 19] identified hydrides of rare-earth elements as potential high-temperature superconductors. Firstprinciples structure searching studies of rare-earth hydrides have reported structures with high hydrogen content adopting cage-like structures [20, 21]. Of particular note, a $T_{c}$ of $264-286 \mathrm{~K}$ was calculated for $F m \overline{3} m$ $\mathrm{LaH}_{10}$ at $210 \mathrm{GPa}$ [20], while the analogous $\mathrm{YH}_{10}$ structure was calculated to have $T_{c}=305-326 \mathrm{~K}$ at $250 \mathrm{GPa}$. Slight distortions of the cubic $\mathrm{LaH}_{10}$ phase were found to lead to $C 2 / m$ and $R \overline{3} m$ structures at lower pressures [22, 23], though Ref. 224] showed that quantum effects render $F m \overline{3} m$ as the true ground state. These predictions were followed by experimental measurement of critical temperatures reaching $260 \mathrm{~K}$ in $\mathrm{LaH}_{10}$ at $170-200$ GPa [25, 26]. The high- $T_{c}$ phase was determined to be a structure with an fcc arrangement of La atoms, lending support to theoretical predictions.

In addition to the aforementioned studies, others have focused on heavier rare-earth hydrides, exploring the synthesis and superconducting properties of cerium [27, 28], praseodymium [29] and neodymium [30] hydrides. Here, within the framework of density functional theory (DFT) 31, 32, we revisit $\mathrm{LaH}_{10}$ and $\mathrm{YH}_{10}$ using crystal structure prediction methods. We find a phase transition to a new hexagonal phase in $\mathrm{LaH}_{10}$ at high pressures, with the metastability of this phase at low pressures offering an explanation for the experimental observation of hcp impurities in fcc samples [26]. We go on to predict the phases and corresponding critical temperatures that may be observed in $\mathrm{YH}_{10}$.

\section{THEORY AND METHODOLOGY}

\section{A. Phonons and superconductivity}

The Hamiltonian of a coupled electron-phonon system 33 can be written as

$$
\begin{gathered}
H=\underbrace{\sum_{k n} \epsilon_{n k} c_{n k}^{\dagger} c_{n k}}_{\text {electronic dispersion }}+\underbrace{\sum_{q \nu} \omega_{q \nu}\left(a_{q \nu}^{\dagger} a_{q \nu}+\frac{1}{2}\right)}_{\text {phonon dispersion }}+ \\
\underbrace{\frac{1}{\sqrt{N_{p}}} \sum_{k q m n \nu} g_{m n \nu}(k, q) c_{m, k+q}^{\dagger} c_{n k}\left(a_{q \nu}+a_{-q \nu}^{\dagger}\right)}_{\text {electron-phonon coupling }} .
\end{gathered}
$$

In this work, we calculate the electronic Kohn-Sham eigenvalues $\epsilon_{n k}$, phonon frequencies $\omega_{q, \nu}$, and electronphonon coupling constants $g_{m n \nu}(k, q)$ appearing in $H$ from first-principles using the QUANTUM ESPRESSO DFT code [34, 35], which we optimised for this work 36. The Hamiltonian in Eq. 14 can be treated within MigdalEliashberg theory [37, allowing us to define the electronboson spectral function

$$
\begin{aligned}
\alpha^{2} F(\omega) & =\frac{1}{N\left(\epsilon_{F}\right)} \sum_{m n q \nu} \delta\left(\omega-\omega_{q \nu}\right) \sum_{k}\left|g_{m n \nu}(k, q)\right|^{2} \\
& \times \delta\left(\epsilon_{m, k+q}-\epsilon_{F}\right) \delta\left(\epsilon_{n, k}-\epsilon_{F}\right) .
\end{aligned}
$$

\footnotetext{
* ams277@cam.ac.uk

$\dagger$ mjh261@cam.ac.uk
} 


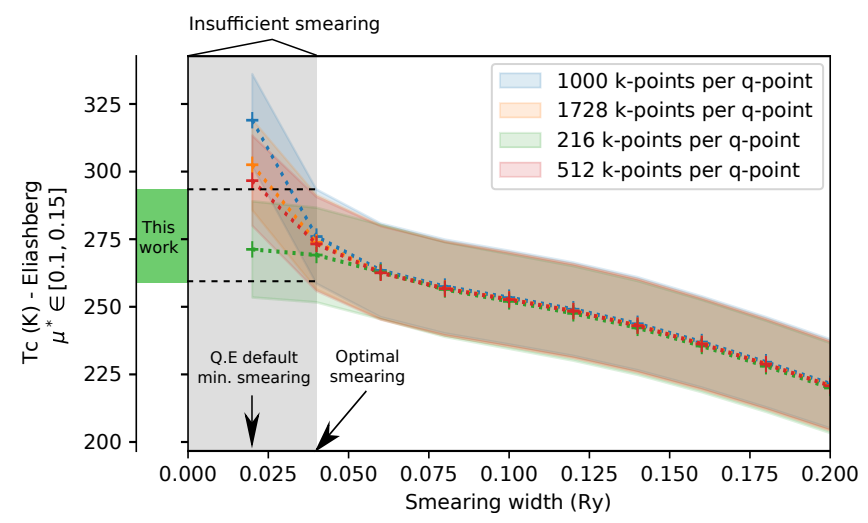

FIG. 1. The dependence of $T_{c}$ on the double-delta smearing width, $\sigma$, for $F m \overline{3} m-\mathrm{YH}_{10}$ at $350 \mathrm{GPa}$. The region of insufficient smearing is shown, along with our choice of $\sigma$ for this structure and pressure. The smallest value used in an electron-phonon calculation with default QUANTUM ESPRESSO settings is shown.

From $\alpha^{2} F$ we extract the superconducting critical temperature by solution of the Eliashberg equations using the ELK code 38. From the quantities appearing in $H$ we may also construct the electronic and vibrational densities of states, from which we can derive the Gibbs free energy as a function of temperature. We do this at a range of pressures, allowing us to construct pressuretemperature phase diagrams.

To evaluate the double-delta sum in Eq. 2 for finite $\mathbf{k}$ and q-point grids, we follow the method detailed in Appendix A of Ref. [39] and smear the delta functions with finite-width Gaussians. In order to best approximate the delta functions, the smallest sensible smearing should be used. However, the smearing must be large enough to accommodate the finite $\mathbf{k}$-point grids used. We identify the optimal choice of smearing from discrepancies in the results between different k-point grids [39], as can be seen in Figs. 1 and 2 ,

Our electron-phonon calculations were carried out using the Perdew-Burke-Ernzerhof (PBE) generalised gradient approximation [40] and ultrasoft pseudopotentials, validated against the all-electron WIEN2k code [41, 42]. Well-converged k-point grids with a spacing of at most $2 \pi \times 0.015 \AA^{-1}$ and an $820 \mathrm{eV}$ plane wave cut-off were used [42]. The q-point grids used were typically 8 times smaller than the k-point grids and were Fourier interpolated to 10 times their original size. For the cubic systems studied, this corresponds to $\geq 24 \times 24 \times 24 \mathbf{k}$-point grids and a $3 \times 3 \times 3$ q-point grid Fourier-interpolated to $30 \times 30 \times 30$.

\section{B. Structure searching}

Our structure searching calculations were performed using $a b$ initio random structure searching (AIRSS) [14, 45] and CASTEP [46. The PBE functional, CASTEP QC5

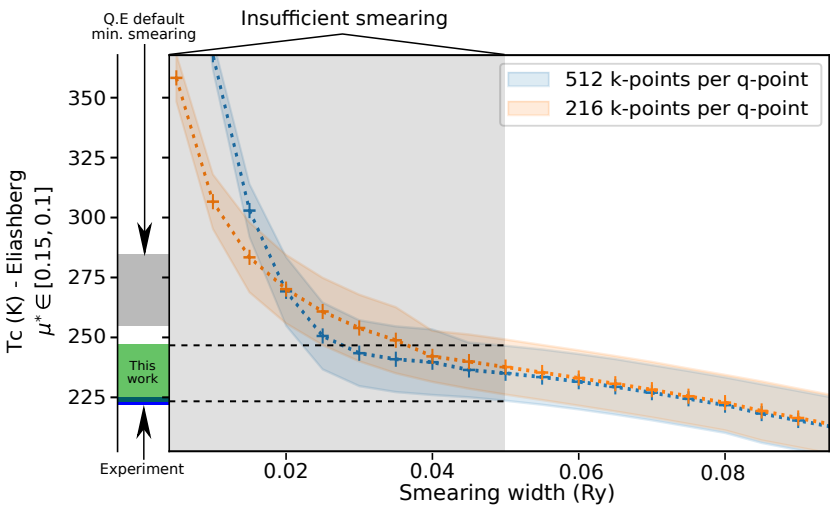

FIG. 2. The dependence of $T_{c}$ on the double-delta smearing width, $\sigma$, for $I m \overline{3} m-\mathrm{YH}_{6}$ at $160 \mathrm{GPa}$. A recent experimental measurement at $166 \mathrm{GPa}$, falling just within our calculated $T_{c}$ range, is also shown [43. We note that Refs. 43, 44] highlighted that previous calculated $T_{c}$ values were considerably higher than their experimental observations and that the results of Ref. 19, which used accurate Wannier interpolation techniques, are in agreement with ours.

pseudopotentials, a $400 \mathrm{eV}$ plane wave cut-off and a $\mathbf{k}$ point spacing of $2 \pi \times 0.05 \AA^{-1}$ were used in these searches unless otherwise stated. The C2X software [47] was used for converting between CASTEP and QUANTUM ESPRESSO file formats, and also for reporting the space groups of structures at various tolerances.

\section{RESULTS AND DISCUSSION}

In the following sections, we report results in terms of phonon-corrected pressures, obtained by fitting the Birch-Murnaghan equation of state [48 to our data. Where static DFT pressures are reported instead, they are labelled as $P_{D F T}$ - this second set of pressures facilitates comparison with previous calculations as they are simply an input parameter to the DFT geometry optimisation.

\section{A. $\mathbf{L a H}_{10}$}

Low enthalpy candidates found by AIRSS for $\mathrm{LaH}_{10}$ include the space groups $F m \overline{3} m, R \overline{3} m$, and a 2-formulaunit $C 2 / \mathrm{m}$, which had been identified previously. The searches also revealed a new structure of $P 6_{3} / m m c$ symmetry. These structures are shown in Fig. 3 A $C 2 / m$ structure with 3 formula units per unit cell was also found to be energetically competitive, but was not considered further in this work as it behaves similarly to the 2formula unit phase over the pressure range of interest. We also found several previously unreported structures at low pressures with space groups Pnnm, $C 2$ and $P 2_{1} 2_{1} 2_{1}$ and unit cells containing 2,3 and 4 formula units, respectively. These are the lowest enthalpy structures in 


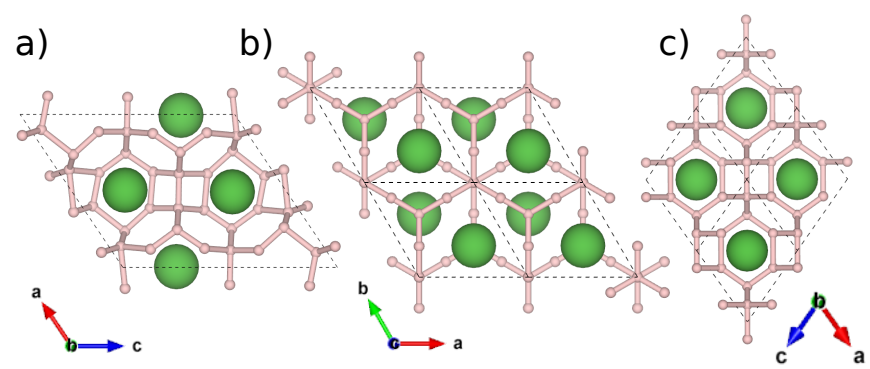

FIG. 3. Structures of $\mathrm{LaH}_{10}$. (a) 2 formula unit/cell $C 2 / m$, (b) 2 formula unit/cell $P 6_{3} / m m c$, (c) 1 formula unit/cell $F m \overline{3} m$. The $R \overline{3} m$ structure is not shown as it is visually indistinguishable from the $F m \overline{3} m$ structure at the pressures of interest.

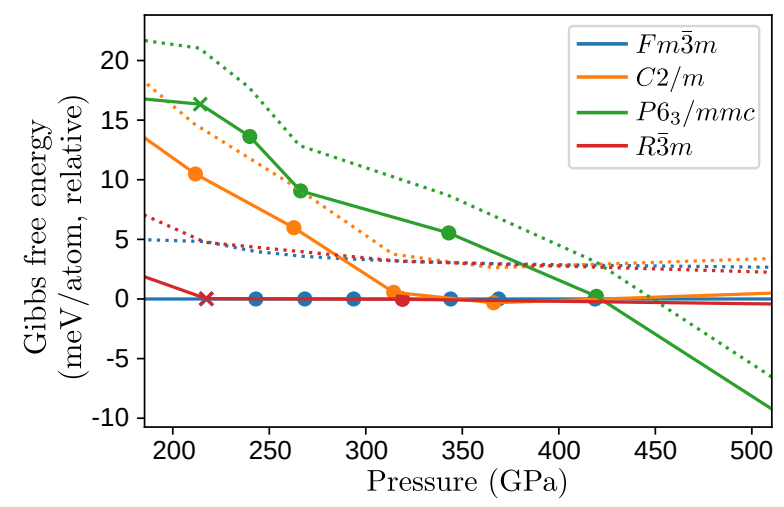

FIG. 4. The Gibbs free energy as a function of pressure for energetically competitive phases of $\mathrm{LaH}_{10}$, plotted relative to a third-order Birch-Murnaghan fit of the $F m \overline{3} m$ data. Crosses represent calculations with unstable phonon modes - these points are not included in the Gibbs free energy fit. Solid lines are at $300 \mathrm{~K}$, dashed lines are at $0 \mathrm{~K}$.

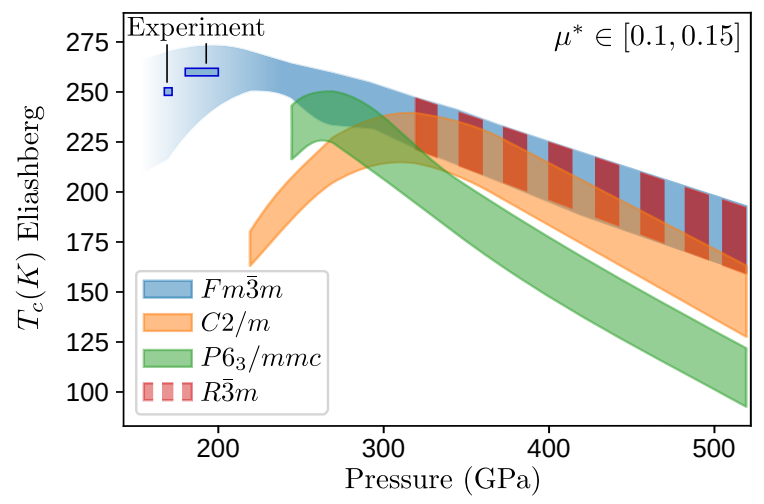

FIG. 5. Calculated $T_{c}(P)$ for dynamically stable phases of $\mathrm{LaH}_{10}$ from direct solution of the Eliashberg equations. The width of the lines arises from our treatment of the MorelAnderson pseudopotential, $\mu^{*}$, 49] as an empirical parameter with values between 0.1 and 0.15 . The $F m \overline{3} m$ result has been extended into the region where it is dynamically unstable (shaded according to unstable fraction of the phonon density of states) in order to facilitate comparison with the experimental results of Refs. 25, 26. This extension was achieved by removing the contribution of unstable phonon modes, in their entirety, to the Eliashberg function while maintaining its normalisation.

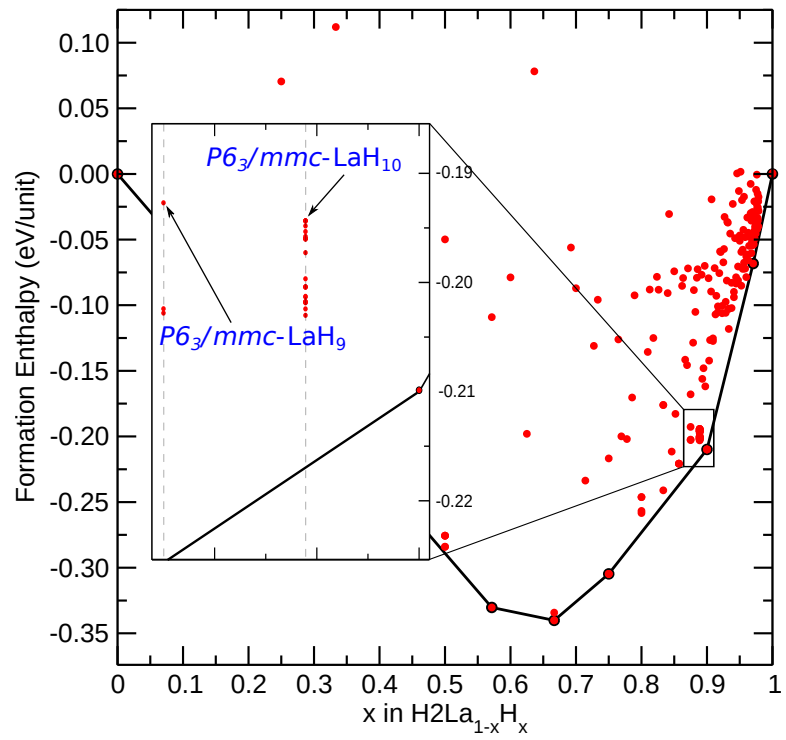

FIG. 6. A convex hull for the La-H system at $150 \mathrm{GPa}$, accurately calculated using CASTEP, k-point spacing of $2 \pi \times 0.03$ $\AA^{-1}$ and a $700 \mathrm{eV}$ plane-wave cut-off. The on-the-fly pseudopotential strings used are provided in the supplementary material 42; the inclusion of a fraction of a $4 \mathrm{f}$ electron in the generation of the La pseudopotential was found to be crucial. A pseudopotential without this addition was unable to reproduce the all-electron $F m \overline{3} m$ - $\mathrm{LaH}_{10} \mathrm{PV}$ curve and led to a qualitatively different convex hull [42. In agreement with Ref. [50], we find that $\mathrm{LaH}_{9}$ is not on the hull at this pressure. However, we also find that $\mathrm{LaH}_{16}$ does not lie on the hull at $150 \mathrm{GPa}$, despite finding the $\mathrm{P} 6 / \mathrm{mmm}-\mathrm{LaH}_{16}$ structure studied in that work.

the low pressure region 42. However, we note that these structures are distortions of the high-symmetry $F m \overline{3} m$ structure and, similarly to the case of $R \overline{3} m$ noted in Ref. [24, it is possible that anharmonic effects may remove them from the potential energy surface. In addition to this, the low symmetry and large unit cells of these structures make converged phonon calculations prohibitively expensive; they are therefore not considered further in this work.

The calculated $\mathrm{LaH}_{10}$ phase behaviour is shown in Fig. 4 and the corresponding critical temperatures are shown in Fig. 5. Our calculations for the $F m \overline{3} m$ phase include unstable phonon modes for $P_{D F T} \leq 210 \mathrm{GPa}$. In the harmonic picture, explicitly taking into account this dynamical instability leads to a window of stability for the $C 2 / m$ phase [42, which is in agreement with previous calculations [22, 23]. However, we note that under the assumption that the unstable modes can be neglected in the calculation of the Gibbs free energy, we obtain the same behaviour as the anharmonic calculations of Ref. [24, i.e., $F m \overline{3} m$ is the only phase with a predicted region of stability at lower pressures. With increasing pressure, as noted in previous theoretical work [24, the $R \overline{3} m$ structure approaches $F m \overline{3} m$ symmetry. We therefore expect that these phases will not be distinguishable at high 
pressures.

At $300 \mathrm{~K}$, the $P 6_{3} / m m c$ structure becomes thermodynamically favourable at pressures above $\sim 420 \mathrm{GPa}$. More importantly, this hexagonal phase is also metastable at low pressures, lying within $20 \mathrm{meV} /$ atom of the cubic phase down to $150 \mathrm{GPa}$, and therefore provides an explanation for the experimental observation of hcp impurities in fcc- $\mathrm{LaH}_{10}$ samples at $170 \mathrm{GPa}$ in Ref. [26.

A low-energy hexagonal $\mathrm{LaH}_{9}$ structure predicted previously in similar pressure regions [50] could offer an alternative explanation for the observation of these impurities. However, the authors of Ref. 26] determined that the two kinds of hcp impurities in their fcc- $\mathrm{LaH}_{10}$ samples possessed $\mathrm{LaH}_{10}$ stoichiometry. We also calculated a high-quality La-H convex hull at $150 \mathrm{GPa}$ using AIRSS 45 and qhull 51 (see Fig. 6). It shows that the $P 6_{3} / m m c-\mathrm{LaH}_{10}$ structure predicted in this work lies closer to the hull than the $P 6_{3} / m m c-\mathrm{LaH}_{9}$ structure of Ref. [50]. It is therefore likely that the hcp impurities originate from our new $P 6_{3} / m m c-\mathrm{LaH}_{10}$ phase.

To facilitate comparison with experiment, we have calculated powder X-ray diffraction patterns for cubic and hexagonal $\mathrm{LaH}_{10}$ and hexagonal $\mathrm{LaH}_{9}$ at $150 \mathrm{GPa}$ - we supply these, alongside the calculated c/a ratios and volumes in the supplementary material [42].

We calculate $T_{c}=232-259 \mathrm{~K}$ for $F m \overline{3} m-\mathrm{LaH}_{10}$ at 269 $\mathrm{GPa}\left(P_{D F T}=250 \mathrm{GPa}\right)$, which is lower than the previous theoretical result of $T_{c}=257-274 \mathrm{~K}$ [20]. However, we observe an increase in $T_{c}$ on reduction of the doubledelta smearing parameter to below our calculated optimal value 42, potentially explaining this discrepancy. Careful choice of smearing has previously been noted as important in other hydride systems [52]. We also note a previous calculation of $T_{c}$ for this structure at 200 GPa [21, however, in agreement with other calculations [22, 23. we find $F m \overline{3} m$ to be dynamically unstable at this pressure. This dynamical instability means we cannot directly compare with experiment, which found $T_{c}$ $=250 \mathrm{~K}$ at around $170 \mathrm{GPa}\left[26\right.$ and $T_{c}=260 \mathrm{~K}$ at $180-200 \mathrm{GPa}$ [25]. However, ignoring the contribution of the unstable phonon modes to the Eliashberg function at pressures $\leq 210 \mathrm{GPa}$ allows for a rough estimation of $T_{c}$ in these regions; this is depicted as the faded-out section in Fig. 5 and the results obtained are in agreement with experimental results. For the $C 2 / m$ phase, using an optimal value of smearing we calculate $T_{c}=205-225 \mathrm{~K}$ at $262 \mathrm{GPa}\left(P_{D F T}=250 \mathrm{GPa}\right)$, compared to $T_{c}=229-245$ $\mathrm{K}$ in Ref. 23].

\section{B. $\mathbf{Y H}_{10}$}

Low-enthalpy candidates for $\mathrm{YH}_{10}$ found using AIRSS include $F m \overline{3} m$, which had been identified previously, a slight distortion of this phase, $R \overline{3} \mathrm{~m}$, and structures of $P 6_{3} / m m c$ and $C m c m$ symmetry. These structures are shown in Fig. 7. The calculated $\mathrm{YH}_{10}$ phase behaviour is shown in Fig. 8 and the corresponding critical tem-
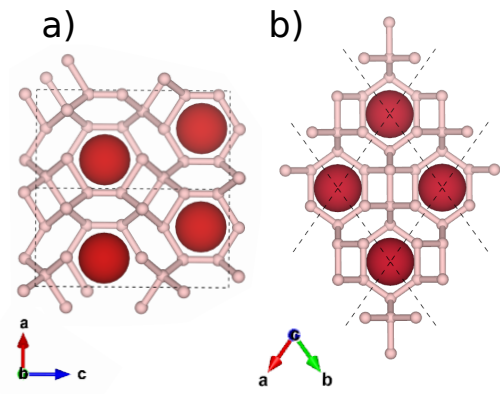

C)

FIG. 7. Structures of $\mathrm{YH}_{10}$. (a) 2 formula unit/cell Cmcm, (b) 1 formula unit/cell $F m \overline{3} m$, (c) 2 formula unit/cell $P 6_{3} / m m c$. The $R \overline{3} m$ structure is, again, not shown.

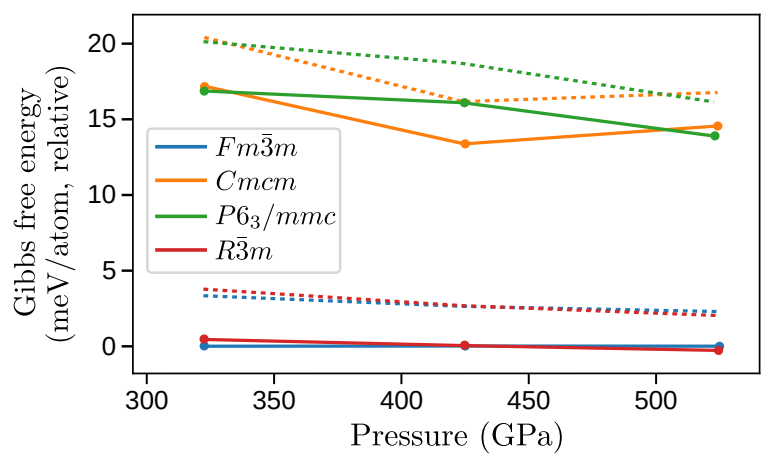

FIG. 8. The Gibbs free energy as a function of pressure for energetically competitive phases of $\mathrm{YH}_{10}$, plotted and interpolated relative to a third-order Birch-Murnaghan fit of the $F m \overline{3} m$ data. Solid lines are at $300 \mathrm{~K}$, dashed lines are at 0 $\mathrm{K}$.

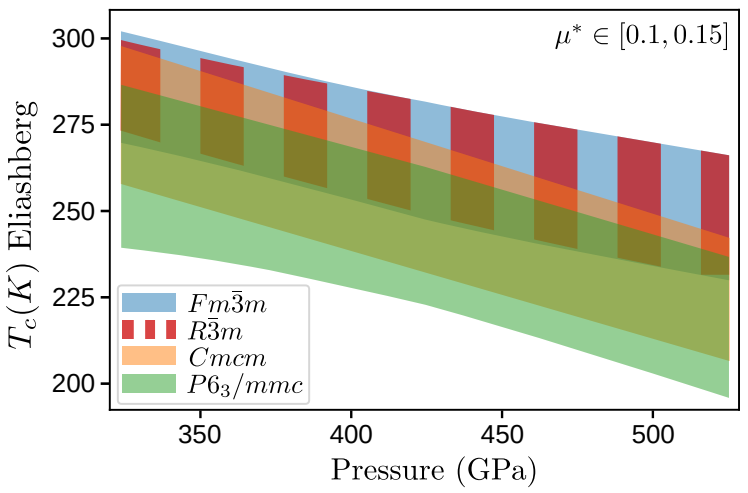

FIG. 9. Calculated $T_{c}(P)$ for dynamically stable phases of $\mathrm{YH}_{10}$ from direct solution of the Eliashberg equations. $\mu^{*}$ is, again, taken to have a value between 0.1 and 0.15 .

peratures are shown in Fig. 9. We do not predict any phase transitions within the predicted range of stability of the $\mathrm{YH}_{10}$ stoichiometry 21. However, the difference in Gibbs free energy between the $F m \overline{3} m$ and $R \overline{3} m$ phases is exceedingly small (see Fig. 8), reflecting their structural similarity.

Previous calculations for $F m \overline{3} m$ found $T_{c}=305-326 \mathrm{~K}$ 
at $250 \mathrm{GPa}$ 20] and $T_{c}=303 \mathrm{~K}$ at $400 \mathrm{GPa}$ [21. Here, we calculate $T_{c}=270-302 \mathrm{~K}$ at $324 \mathrm{GPa}\left(P_{D F T}=300\right.$ $\mathrm{GPa})$ and $T_{c}=250-280 \mathrm{~K}$ at $425 \mathrm{GPa}\left(P_{D F T}=400 \mathrm{GPa}\right)$. Our more conservative $T_{c}$ results can again be explained by considering the smearing parameter used to approximate the double-delta integral in Eq. 2. We were able to reproduce the results of previous calculations by using the minimum default smearing employed in QUANTUM ESPRESSO, which in this case overestimates $T_{c}$ by $\sim 30$ K (see Fig. 1) compared to optimal smearing. We note that our results agree with those obtained using Wannier interpolation techniques [19]. Using the same method to calculate an optimal smearing also provides results in agreement with recent experimental measurements for Im $\overline{3} m-\mathrm{YH}_{6}$ [43, as shown in Fig. 2.

\section{CONCLUSIONS}

We have identified a new hexagonal phase of $\mathrm{LaH}_{10}$ with $P 6_{3} / m m c$ symmetry. Our calculations show a pressure-induced phase transition into this new phase from the cubic phase believed to be observed in experiment [25, 26]. The overall phase behaviour predicted within the harmonic picture is $C 2 / m \rightarrow F m \overline{3} m \rightarrow$ $P 6_{3} / m m c$ with all three of these phases predicted to be high- $T_{c}$ superconductors. Making the assumption that unstable modes can be neglected, however, gives the same picture as the anharmonic calculations of Ref. 24] where $F m \overline{3} m$ is the true ground state at lower pressures. The new hexagonal phase predicted here offers a direct explanation for the observation of hcp impurities in recent experiments [26].

We found that $\mathrm{YH}_{10}$ adopts very similar structures to
$\mathrm{LaH}_{10}$, with one of $P 6_{3} / m m c$ symmetry again amongst the most energetically competitive candidates. Over the pressure range considered the $F m \overline{3} m / R \overline{3} m$ phase remains the most stable. The difference in Gibbs free energy between these two structures is extremely small, meaning synthesis of a pure sample of either could be difficult.

We found the double-delta smearing employed in superconductivity calculations to be of particular importance. Its effect on calculated $T_{c}$ changes from system to system; in particular, in our calculations the default minimum smearing employed by QUANTUM ESPRESSO overestimates $T_{c}$ for $\mathrm{LaH}_{10}$ by $\sim 20 \mathrm{~K}$ and $\mathrm{YH}_{10}$ by $\sim 30 \mathrm{~K}$ when compared to optimal smearing.

\section{ACKNOWLEDGEMENTS}

We thank Bartomeu Monserrat for helpful discussions. A.M.S. acknowledges funding through an EPSRC studentship. M.J.H. and M.S.J. acknowledge the EPSRC Centre for Doctoral Training in Computational Methods for Materials Science for funding under grant number EP/L015552/1. C.J.P. is supported by the Royal Society through a Royal Society Wolfson Research Merit award. R.J.N. is supported by EPSRC under Critical Mass Grant EP/P034616/1 and the UKCP consortium grant EP/P022596/1. This work was performed using resources provided by the Cambridge Service for Data Driven Discovery (CSD3) operated by the University of Cambridge Research Computing Service ( www.csd3.cam.ac.uk), provided by Dell EMC and Intel using Tier-2 funding from the EPSRC (capital grant $\mathrm{EP} / \mathrm{P} 020259 / 1)$, and DiRAC funding from the STFC (www.dirac.ac.uk).
[1] N. W. Ashcroft, Phys. Rev. Lett. 21, 1748 (1968).

[2] J. M. McMahon, M. A. Morales, C. Pierleoni, and D. M. Ceperley, Rev. Mod. Phys 84, 1607 (2012).

[3] H.-K. Mao and R. J. Hemley, Science 244, 1462 (1989).

[4] M. Eremets and I. Troyan, Nature Materials 10, 927 (2011).

[5] P. Dalladay-Simpson, R. T. Howie, and E. Gregoryanz, Nature 529, 63 (2016).

[6] R. P. Dias and I. F. Silvera, Science 355, 715 (2017).

[7] P. Loubeyre, F. Occelli, and P. Dumas, Nature 577, 631 (2020).

[8] N. W. Ashcroft, Phys. Rev. Lett. 92, 187002 (2004).

[9] D. Duan, Y. Liu, Y. Ma, Z. Shao, B. Liu, and T. Cui, Natl. Sci. Rev 4, 121 (2017).

[10] E. Zurek and T. Bi, J. Chem. Phys. 150, 050901 (2019).

[11] J. A. Flores-Livas, L. Boeri, A. Sanna, G. Profeta, R. Arita, and M. Eremets, Physics Reports (2020), https://doi.org/10.1016/j.physrep.2020.02.003.

[12] L. Boeri and G. B. Bachelet, J. Phys: Condens. Matt. 31, 234002 (2019).
[13] A. R. Oganov, C. J. Pickard, Q. Zhu, and R. J. Needs, Nature Reviews Materials 4, 331 (2019).

[14] R. J. Needs and C. J. Pickard, APL Materials 4, 053210 (2016).

[15] C. J. Pickard, I. Errea, and M. I. Eremets, Annual Review of Condensed Matter Physics 11, 57 (2020).

[16] A. P. Durajski and R. Szczesniak, Supercond. Sci. and Tech. 27, 115012 (2014).

[17] D. Y. Kim, R. H. Scheicher, and R. Ahuja, Phys. Rev. Lett. 103, 077002 (2009).

[18] Y. Li, J. Hao, H. Liu, J. S. Tse, Y. Wang, and Y. Ma, Scientific Reports 5, 9948 (2015).

[19] C. Heil, S. di Cataldo, G. B. Bachelet, and L. Boeri, Phys. Rev. B 99, 220502(R) (2019).

[20] H. Liu, I. I. Naumov, R. Hoffmann, N. W. Ashcroft, and R. J. Hemley, Proc. Nat. Acad. Sci. 114, 6990 (2017).

[21] F. Peng, Y. Sun, C. J. Pickard, R. J. Needs, Q. Wu, and Y. Ma, Phys. Rev. Lett. 119, 107001 (2017).

[22] Z. M. Geballe, H. Liu, A. K. Mishra, M. Ahart, M. Somayazulu, Y. Meng, M. Baldini, and R. J. Hemley, Angewandte Chemie International Edition 57, 688 (2018). 
[23] H. Liu, I. I. Naumov, Z. M. Geballe, M. Somayazulu, J. S. Tse, and R. J. Hemley, Phys. Rev. B 98, 100102(R) (2018).

[24] I. Errea, F. Belli, L. Monacelli, A. Sanna, T. Koretsune, T. Tadano, R. Bianco, M. Calandra, R. Arita, F. Mauri, and J. A. Flores-Livas, Nature 578, 66 (2020).

[25] M. Somayazulu, M. Ahart, A. K. Mishra, Z. M. Geballe, M. Baldini, Y. Meng, V. V. Struzhkin, and R. J. Hemley, Phys. Rev. Lett. 122, 027001 (2019).

[26] A. P. Drozdov, P. P. Kong, V. S. Minkov, S. P. Besedin, M. A. Kuzovnikov, S. Mozaffari, L. Balicas, F. F. Balakirev, D. E. Graf, V. B. Prakapenka, E. Greenberg, D. A. Knyazev, M. Tkacz, and M. I. Eremets, Nature 569, 528 (2019).

[27] N. P. Salke, M. M. D. Esfahani, Y. Zhang, I. A. Kruglov, J. Zhou, Y. Wang, E. Greenberg, V. B. Prakapenka, J. Liu, A. R. Oganov, and J.-F. Lin, Nature Communications 10, 1 (2019).

[28] X. Li, X. Huang, D. Duan, C. J. Pickard, D. Zhou, H. Xie, Q. Zhuang, Y. Huang, Q. Zhou, B. Liu, and T. Cui, Nature Communications 10, 3461 (2019).

[29] D. Zhou, D. V. Semenok, D. Duan, H. Xie, W. Chen, X. Huang, X. Li, B. Liu, A. R. Oganov, and T. Cui, Science Advances 6 (2020), 10.1126/sciadv.aax6849, https://advances.sciencemag.org/content/6/9/eaax6849.

[30] D. Zhou, D. V. Semenok, H. Xie, X. Huang, D. Duan, A. Aperis, P. M. Oppeneer, M. Galasso, A. I. Kartsev, A. G. Kvashnin, A. R. Oganov, and T. Cui, Journal of the American Chemical Society 142, 2803 (2020)

[31] P. Hohenberg and W. Kohn, Phys. Rev 136, B864 (1964).

[32] W. Kohn and L. J. Sham, Phys. Rev 140, A1133 (1965).

[33] F. Giustino, Rev. Mod. Phys. 89, 015003 (2017)

[34] P. Giannozzi, S. Baroni, N. Bonini, M. Calandra, R. Car, C. Cavazzoni, D. Ceresoli, G. L. Chiarotti, M. Cococcioni, I. Dabo, A. Dal Corso, S. de Gironcoli, S. Fabris, G. Fratesi, R. Gebauer, U. Gerstmann, C. Gougoussis, A. Kokalj, M. Lazzeri, L. Martin-Samos, N. Marzari, F. Mauri, R. Mazzarello, S. Paolini, A. Pasquarello, L. Paulatto, C. Sbraccia, S. Scandolo, G. Sclauzero, A. P. Seitsonen, A. Smogunov, P. Umari, and R. M. Wentzcovitch, Journal of Physics: Condensed Matter 21, 395502 (19pp) (2009).

[35] P. Giannozzi, O. Andreussi, T. Brumme, O. Bunau, M. B. Nardelli, M. Calandra, R. Car, C. Cavazzoni, D. Ceresoli, M. Cococcioni, N. Colonna, I. Carnimeo, A. D. Corso, S. de Gironcoli, P. Delugas, R. A. DiStasio, A. Ferretti, A. Floris, G. Fratesi, G. Fugallo, R. Gebauer, U. Gerstmann, F. Giustino, T. Gorni, J. Jia, M. Kawamura, H.-Y. Ko, A. Kokalj, E. Küçükbenli, M. Lazzeri, M. Marsili, N. Marzari, F. Mauri, N. L. Nguyen, H.V. Nguyen, A. O. de-la Roza, L. Paulatto, S. Poncé, D. Rocca, R. Sabatini, B. Santra, M. Schlipf, A. P. Seitsonen, A. Smogunov, I. Timrov, T. Thonhauser, P. Umari, N. Vast, X. Wu, and S. Baroni, Journal of Physics: Condensed Matter 29, 465901 (2017).

[36] Our optimisations have been submitted to the QUANTUM ESPRESSO project.

[37] G. M. Eliashberg, Sov. Phys. JETP 11, 696 (1960).

[38] http://elk.sourceforge.net/, the ELK FP-LAPW code.

[39] M. Wierzbowska, S. de Gironcoli, and P. Giannozzi, arXiv:cond-mat/0504077 (2005).

[40] J. P. Perdew, K. Burke, and M. Ernzerhof, Phys. Rev. Lett. 77, 3865 (1996).
[41] P. Blaha, K. Schwarz, G. K. Madsen, D. Kvasnicka, and J. Luitz, http://www.wien2k.at/reg_user/textbooks/ usersguide.pdf/ (2001), wien2k user guide.

[42] See supplementary information.

[43] I. A. Troyan, D. V. Semenok, A. G. Kvashnin, A. V. Sadakov, O. A. Sobolevskiy, V. M. Pudalov, A. G. Ivanova, V. B. Prakapenka, E. Greenberg, A. G.Gavriliuk, V. V. Struzhkin, A. Bergara, I. Errea, R. Bianco, M. Calandra, F. Mauri, L. Monacelli, R. Akashi, and A. R. Oganov, arXiv preprint arXiv:1908.01534 (2020).

[44] P. P. Kong, V. S. Minkov, M. A. Kuzovnikov, S. P. Besedin, A. P. Drozdov, S. Mozaffari, L. Balicas, F. F. Balakirev, V. B. Prakapenka, E. Greenberg, D. A. Knyazev, and M. I. Eremets, arXiv preprint arXiv:1909.10482 (2019).

[45] C. J. Pickard and R. J. Needs, J. Phys: Condens. Matt. 23, 053201 (2011).

[46] S. J. Clark, M. D. Segall, C. J. Pickard, P. J. Hasnip, M. I. J. Probert, K. Refson, and M. C. Payne, Zeitschrift für Kristallographie-Crystalline Materials 220, 567 (2005).

[47] M. Rutter, Computer Physics Communications 225, 174 (2018)

p48 Firch, Phys. Rev. 71, 809 (1947)

[49] P. Morel and P. W. Anderson, Phys. Rev. 125, 1263 (1962).

[50] I. A. Kruglov, D. V. Semenok, H. Song, R. L. Szczesniak, I. A. Wrona, R. Akashi, M. M. D. Esfahani, D. Duan, T. Cui, A. G. Kvashnin, and A. R. Oganov, Phys. Rev. B 101, 024508 (2020).

[51] C. B. Barber, D. P. Dobkin, and H. T. Huhdanpaa, ACM Trans. on Mathematical Software 22, 469 (1996).

[52] C. Heil, G. B. Bachelet, and L. Boeri, Phys. Rev. B 97, 214510 (2018). 


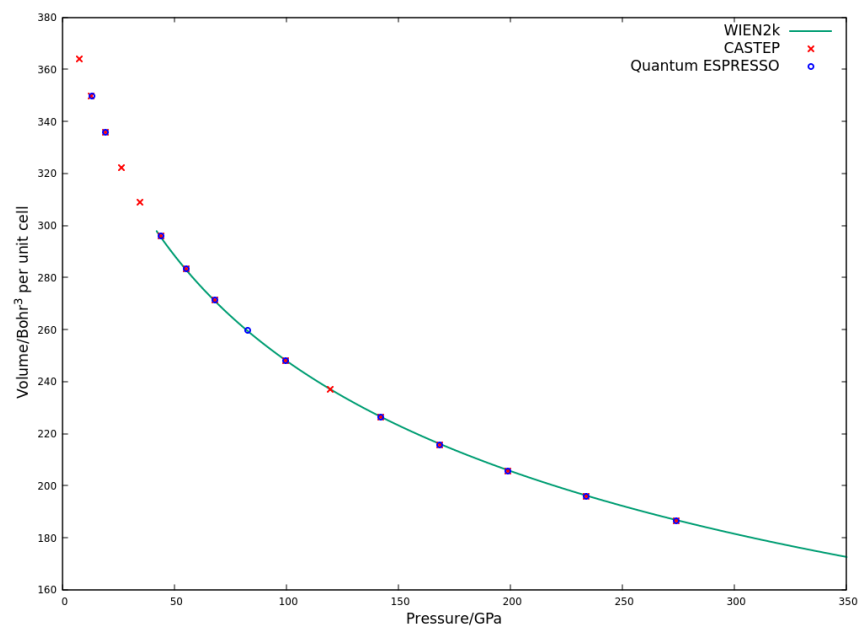

FIG. 10. Pressure-volume curve calculated using the allelectron code WIEN2k compared to data obtained using CASTEP and QUANTUM ESPRESSO for $F m \overline{3} m-\mathrm{LaH}_{10}$. The WIEN2k data was calculated for Ref. [23] and was provided to us by Hanyu Liu.

\section{STABILITY AND SUPERCONDUCTIVITY OF LANTHANUM AND YTTRIUM DECAHYDRIDES - SUPPLEMENTARY MATERIAL}

\section{PRESSURE-VOLUME CURVES}

In order to validate the pseudopotentials used in QUANTUM ESPRESSO, we compared the pressure-volume curves produced for the clathrate structures $\mathrm{LaH}_{10}$ and $\mathrm{YH}_{6}$ to data obtained using CASTEP 46 and using the allelectron code WIEN2k 41]. Figs. 10 and 11 contain data points produced by the following codes and pseudopotentials:

- quANTUM ESPREsso [34, 35]: Scalar-relativistic, ultrasoft PBE pseudopotentials downloaded from https://www.quantum-espresso.org/ pseudopotentials/ps-library

- CASTEP 46]: on-the-fly (OTF) pseudopotentials generated with default pseudopotential strings for hydrogen and yttrium and $2|2.3| 5|6| 7 \mid 50 \mathrm{U}: 60: 51: 52: 43\{4 \mathrm{f} 0.1\}(\mathrm{qc}=4.5)[4 \mathrm{f} 0.1]$ for lanthanum - in terms of generating a pseudopotential capable of exactly reproducing the all-electron PV curve for $\mathrm{LaH}_{10}$, we find that the addition of $4 \mathrm{f} 0.1$ to the La pseudopotential string is crucial.

Good agreement between QUANTUM ESPRESSO and WIEN2k is observed in both cases. For the most part, the structure searches in this work used CASTEP QC5 pseudopotentials, instead of OTF pseudopotentials, for computational efficiency. The detailed La-H convex hull at $150 \mathrm{GPa}$ presented in the main text used

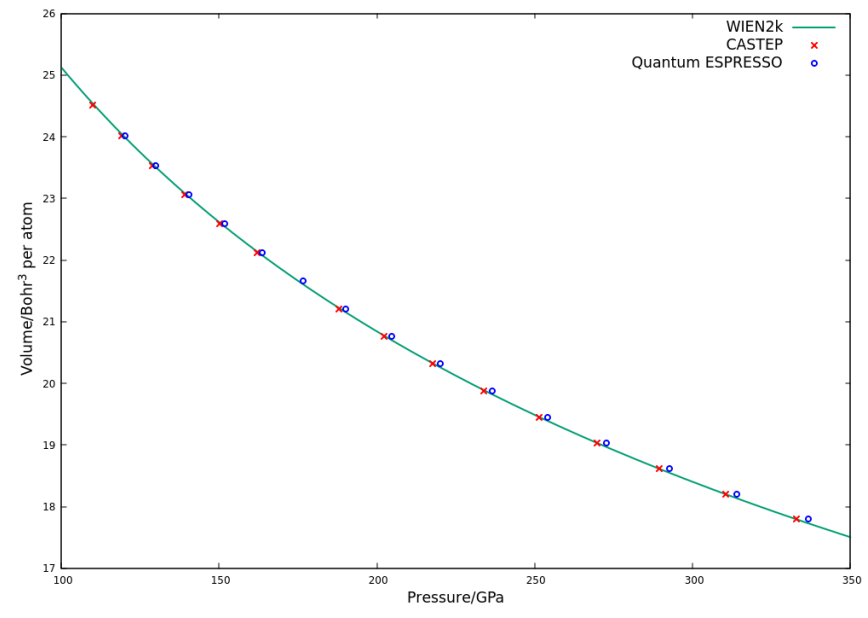

FIG. 11. Pressure-volume curve calculated using the allelectron code WIEN2k compared to data obtained using CASTEP and QUANTUM ESPRESSO for $I m \overline{3} m-\mathrm{YH}_{6}$. The WIEN2k data was calculated for Ref. 21] and was provided to us by Feng Peng.

CASTEP OTF pseudopotentials generated by the string $2|2.3| 5|6| 7 \mid 50 \mathrm{U}: 60: 51: 52: 43\{4 \mathrm{f0} .1\}(\mathrm{qc}=4.5)[4 \mathrm{f} 0.1]$ for La and the default string for $\mathrm{H}$.

\section{STRUCTURE SEARCHING AND CONVEX HULLS}

We constructed well-converged convex hulls for the La$\mathrm{H}$ and Y-H systems using AIRSS [14, 45] and qhull [51, as illustrated in Figs. 12 and 13. Our convex hulls confirm the findings of previous work showing that the $\mathrm{LaH}_{10}$ and $\mathrm{YH}_{10}$ stoichiometries are on or close to the hull over the pressure ranges we study here $\left(\sim 150-500 \mathrm{GPa}\right.$ for $\mathrm{LaH}_{10}$ and $>\sim 300 \mathrm{GPa}$ for $\mathrm{YH}_{10}$ ) 21]. Powder X-ray diffraction patterns for structures relating to hexagonal impurities are shown in Fig. 14. At $150 \mathrm{GPa}$, we find a c/a ratio of 1.526 for $P 6_{3} / m m c-\mathrm{LaH}_{10}$ and of 1.564 for $P 6_{3} / m m c-$ $\mathrm{LaH}_{9}$. At the same pressure, the volume per formula unit for $P 6_{3} / m m c-\mathrm{LaH}_{10}$ is $33.15 \AA^{3}$ and for $P 6_{3} / m m c-\mathrm{LaH}_{9}$ is $31.73 \AA^{3}$.

Throughout this work, we found the $\mathrm{C} 2 \mathrm{x}$ software 47] extremely useful for converting between CASTEP and QUANTUM ESPRESSO file formats and reporting symmetries at various tolerances.

\section{DFT ENERGIES}

Fig. 15 shows the relative energies of the $\mathrm{LaH}_{10}$ phases, neglecting phonon contributions. Fig. 17 shows the same for $\mathrm{YH}_{10}$ phases. The structure files for all structures studied in this work are available at https://doi.org/ 10.17863/CAM. 46481 


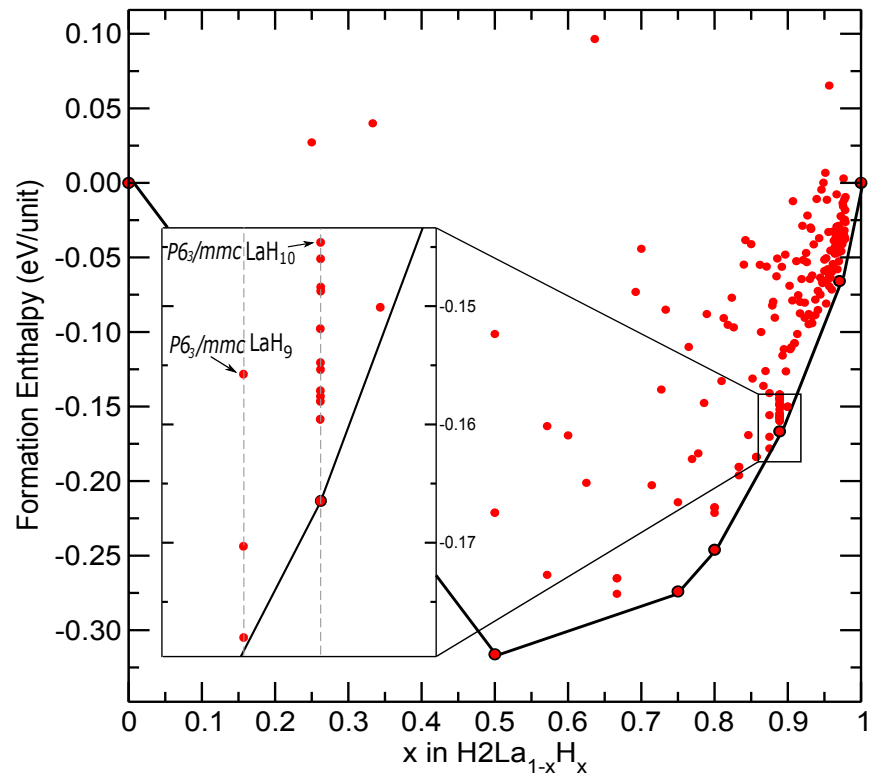

FIG. 12. A convex hull for the La-H system at $150 \mathrm{GPa}$, calculated with the same parameters as the La-H convex hull in the main text, but without the 0.14 f electron in the La pseudopotential. Here, $\mathrm{LaH}_{3}, \mathrm{LaH}_{5}, \mathrm{LaH}_{6}, \mathrm{LaH}_{10}$ and $\mathrm{LaH}_{35}$ are found on the hull, compared to $\mathrm{La}_{3} \mathrm{H}_{10}, \mathrm{LaH}_{4}, \mathrm{LaH}_{5}, \mathrm{LaH}_{11}$ and $\mathrm{LaH}_{35}$ in the hull of the main text, highlighting the importance of the $4 \mathrm{f}$ electron contribution.

\section{CONVERGENCE TESTING}

The first stage towards calculating accurate phase diagrams and superconducting critical temperatures is to establish the computational parameters required to achieve the desired accuracy. In this work, the most relevant parameters were

- Electronic plane-wave cutoff

- Electronic k-point sampling density

- Electronic occupation smearing width/scheme

- Phonon q-point sampling density

The electronic occupation smearing width was found to be of particular importance. It is important to note that this is the Fermi-surface smearing used during SCF convergence, not the smearing used to evaluate double-delta integrals in superconductivity theory. For typical DFT energy calculations the smearing of the electronic Fermi surface often makes a negligible difference, even with high effective temperatures. This is because the energy scale of the electronic band structure is typically equivalent to thousands of Kelvin, due to Fermi statistics pushing electrons into higher and higher energy states [? ]. As the total energies are only sensitive to the average change in the occupied energy states, and because the smearing is symmetric around the Fermi surface, we can use high smearing temperatures when we are only interested in

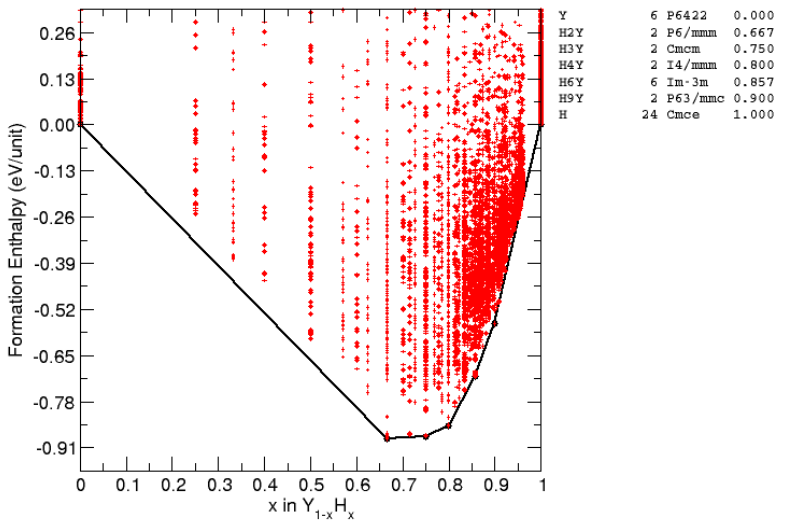

FIG. 13. An example convex hull for the Y-H system, showing that $\mathrm{YH}_{10}$ is meta-stable at the static lattice level at $400 \mathrm{GPa}$. We note that our $\mathrm{Y}-\mathrm{H}$ hull identifies the $\mathrm{YH}_{4}, \mathrm{YH}_{6}$ and $\mathrm{YH}_{9}$ structures recently reported in experiment [43, 44] $(I 4 / \mathrm{mmm}$, Im $\overline{3} m$ and $P 6_{3} / m m c$, respectively), highlighting the success of crystal structure prediction methods.

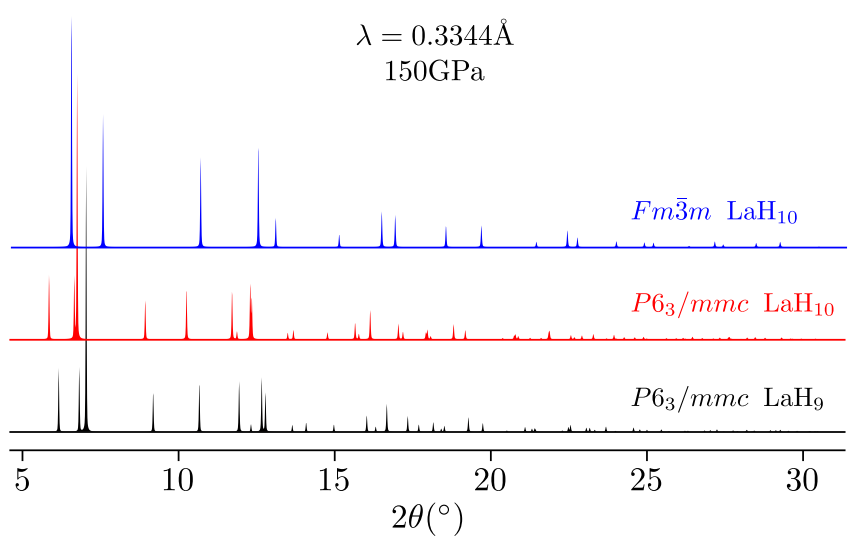

FIG. 14. Simulated X-ray powder diffraction patterns for three of the structures discussed in this work.

total energies. Unfortunately the same is not true for electron-phonon coupling parameters. Only states close to the Fermi surface contribute significantly to electronphonon coupling. Therefore, in order to calculate accurate electron-phonon coupling properties, we need an accurate resolution of the (unsmeared) Fermi surface. To describe a Fermi surface accurately, we therefore require good Brillouin zone resolution, which can be achieved using large k-point grids. This can be seen in Fig. 18. The resulting parameter set for $\mathrm{LaH}_{10}$ is a 60 Ry cutoff, a k-point grid with a spacing of $2 \pi \times 0.015 \AA^{-1}$ (equivalent to a $24 \times 24 \times 24$ grid for the $F m \overline{3} m$ phase) and a q-point grid that is 8 times smaller than the k-point grid (equivalent to a $3 \times 3 \times 3$ grid for the $F m \overline{3} m$ phase). From Fig. 18, we see that the difference between using $300 \mathrm{~K}$ and $3000 \mathrm{~K}$ smearing leads to a greater error in 


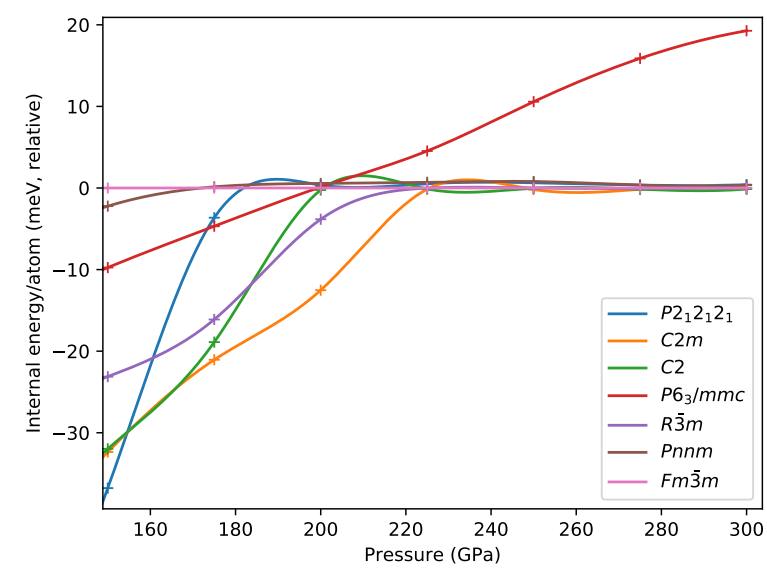

FIG. 15. DFT internal energies of $\mathrm{LaH}_{10}$ phases, neglecting phonon contributions. We see that at lower pressures, several distortions of the $F m \overline{3} m$ phase have lower internal energies we therefore predict that within the harmonic approximation these distorted phases have a range of stability below $\sim 210$ GPa when $F m \overline{3} m$ becomes dynamically unstable. The electronic energy shows that these distortions approach $F m \overline{3} m$ at higher pressures. This behaviour is reflected in the Gibbs free energy plot in the main text and can be observed by considering symmetry tolerances between the structures.

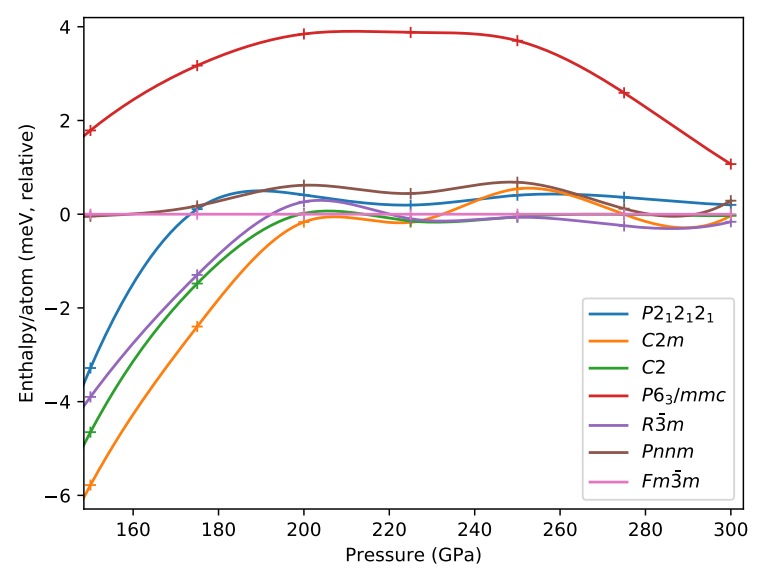

FIG. 16. DFT enthalpies of $\mathrm{LaH}_{10}$ phases, including the largeunit-cell Pnnm, $P 2_{1} 2_{1} 2_{1}$ and $C 2$ phases. We see that these large-cell phases are energetically competitive.

$T_{c}$ than using an approximate $\mu^{*}$, but does not noticeably impact convergence; this allows us to use $300 \mathrm{~K}$ smearing without significant loss of efficiency. In order to carry out these calculations within a reasonable timeframe, we have optimised the electron-phonon coupling code in QUANTUM ESPRESSO, leading to a $10 \times$ speedup for our systems. These changes have been submitted (and accepted) to the QUANTUM ESPRESSO project to allow others to benefit from our modifications. Since the
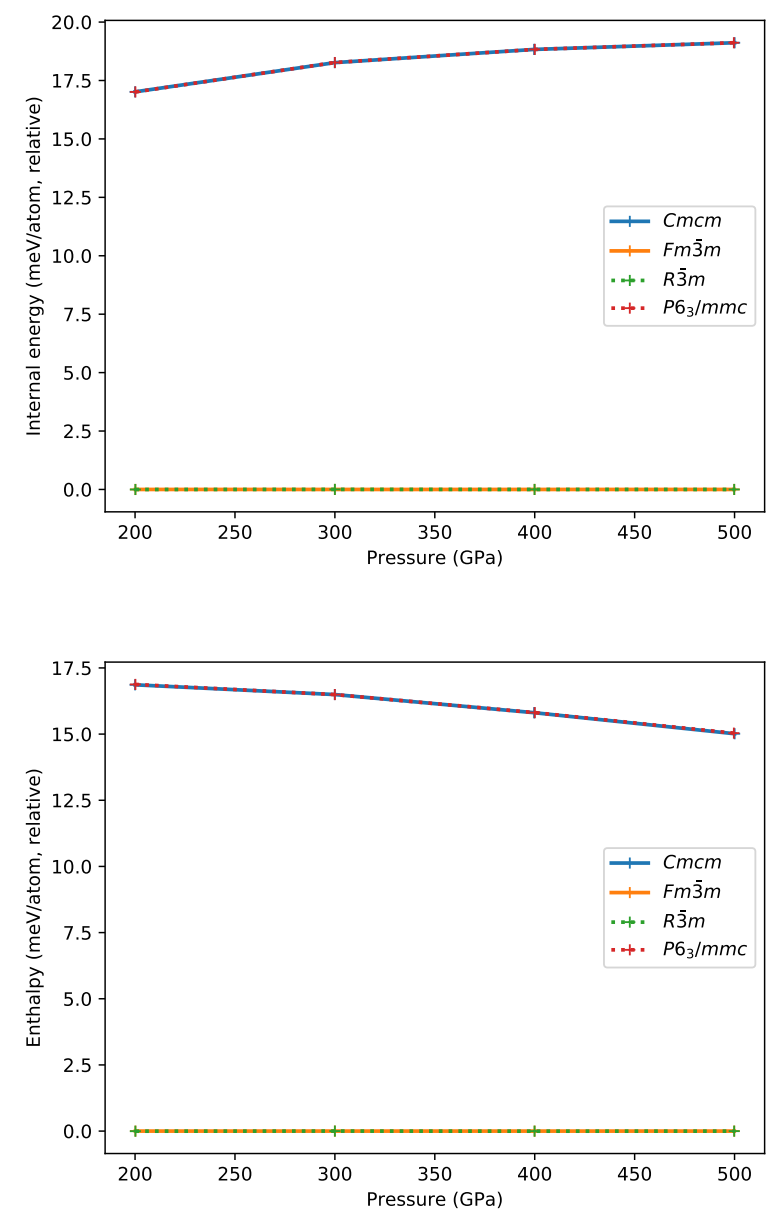

FIG. 17. DFT energetics of $\mathrm{YH}_{10}$ phases, neglecting phonon contributions. Top panel: internal energy. Bottom panel: enthalpy. By considering symmetry tolerances between the structures of interest and studying the internal energy, it is clear that the $F m \overline{3} m$ and $R \overline{3} m$ structures are very similar, as are the $P 6_{3} / \mathrm{mmc}$ and $\mathrm{Cmcm}$ structures. The energy difference between the two sets of structures at the static-lattice level is too large to be compensated for by differences in phonon contributions to the energy and we do not see any region of stability for $P 6_{3} / \mathrm{mmc}$ or $\mathrm{Cmcm}$ over the pressure range of interest, as can be seen in the Gibbs free energy plot in the main text.

electron-phonon calculations require such high convergence parameters, thermodynamic quantities are already well-converged with the chosen parameters, as shown in Fig. 19 and 20

\section{TREATMENT OF DOUBLE-DELTA SMEARING}

As noted in the main text, we use a multiple-grid scheme to ensure that the double-delta smearing parameters used are appropriate. It is straightforward to see 
$\sigma=0.005 \mathrm{Ry}$
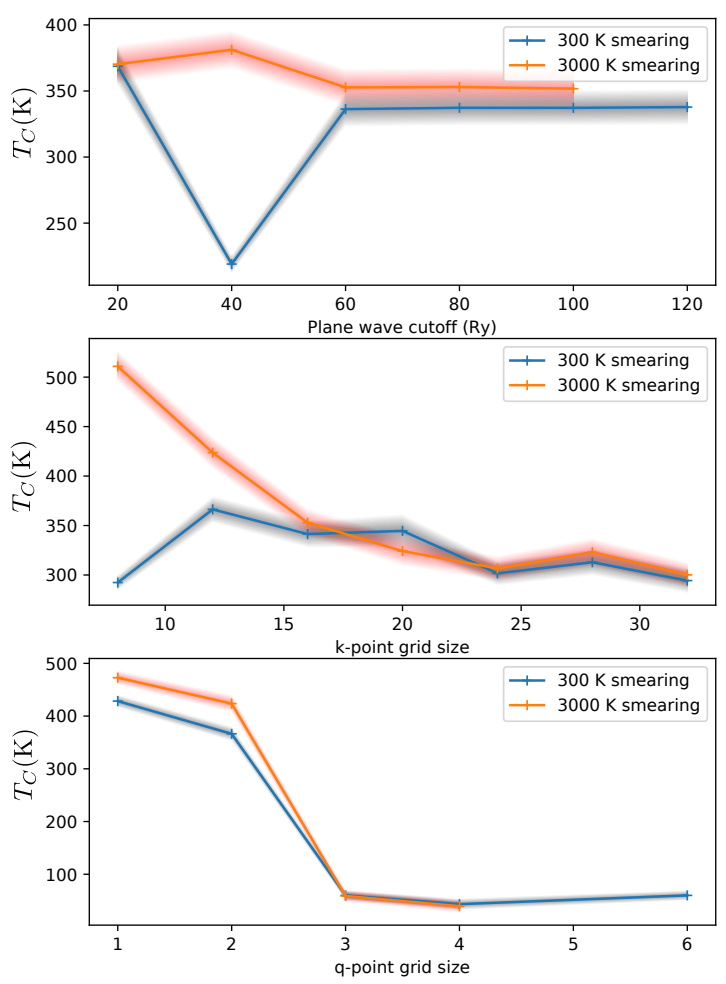

$\sigma=0.025 \mathrm{Ry}$
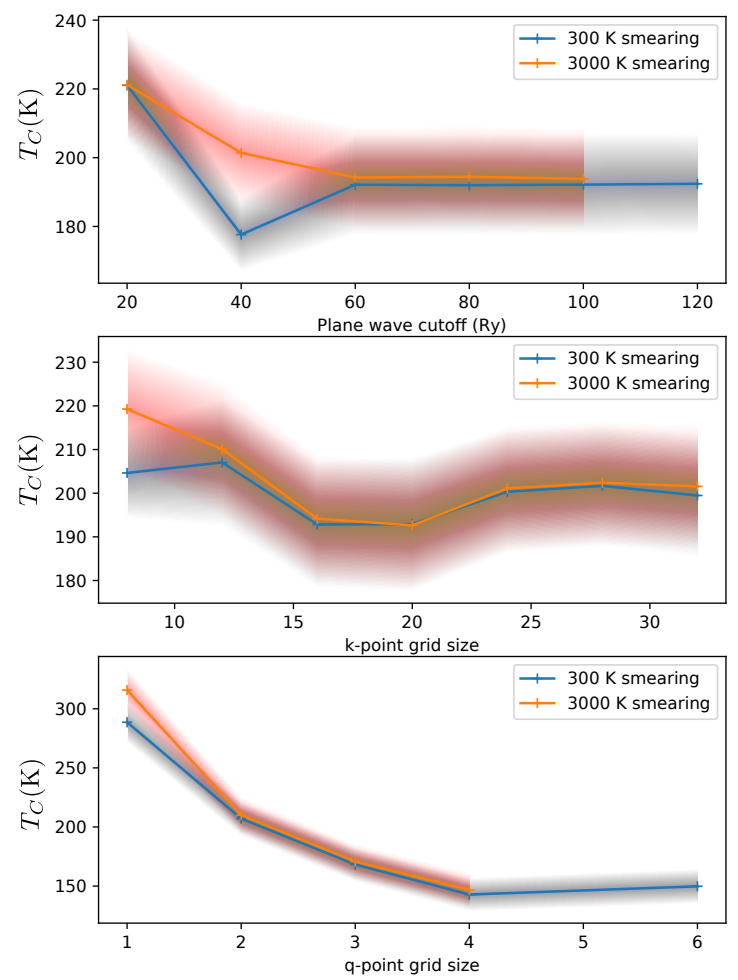

FIG. 18. Convergence properties for the critical temperature of the $F m \overline{3} m$ phase of $\mathrm{LaH}_{10}$ for different values of double-delta smearing $\sigma$. The blurred section around each line represents the distribution of $T_{C}$ for different values of the $\mu^{*}$ parameter.

that below a certain smearing value strong discrepancies between different grid sizes arise; see Fig. 22 for example. We have modified our version of QUANTUM ESPRESSO so that we can increase the number of double-delta smearing values used, which ensures that we can always identify this region of insufficient smearing.

\section{PHONON DISPERSION CURVES}

Fig. 21]shows the phonon dispersion, linewidths and resulting Eliashberg function for the $F m \overline{3} m$ phase of $\mathrm{LaH}_{10}$ at $200 \mathrm{GPa}$. This is the highest pressure at which imaginary phonon modes are present, and where we apply our procedure for estimating $T_{C}$ in the presence of imaginary modes.

\section{BACKGROUND THEORY: ELECTRON-PHONON COUPLING IN DFT}

Typically, within DFT the nuclear coordinates, $R$, are treated as fixed and the electronic Kohn-Sham system is solved within the fixed nuclear potential. In order to calculate the effects of electron-phonon coupling within the DFT formalism we must consider leading-order corrections to the Born-Oppenheimer approximation in nuclear displacements. Expanding our Kohn-Sham potential in terms of these displacements leads to

$$
V_{K S}(R+\delta R)=V_{K S}(R)+\sum_{\kappa, p} \frac{\partial V_{K S}}{\partial R_{\kappa, p}} \cdot \delta R_{\kappa, p}+O\left(\delta R^{2}\right) .
$$

where $R_{\kappa, p}$ is the position of atom $\kappa$ in unit cell $p$. An atomic displacement of an atom can be written in terms of phonon creation and annihilation operators [33] as

$$
\delta R_{\kappa, p}=\frac{1}{\sqrt{N_{p} M_{\kappa}}} \sum_{q \nu} e^{i q \cdot R_{p}} \frac{1}{\sqrt{2 \omega_{q \nu}}}\left(a_{q \nu}+a_{-q \nu}^{\dagger}\right) e_{\kappa \nu}(q)
$$

where $e_{k \nu}(q)$ and $\omega_{q, \nu}$ are, respectively, the eigenvector and frequency of the phonon mode with creation operator $a_{q \nu}^{\dagger} . \quad R_{p}$ is the position of the $p^{\text {th }}$ unit cell within the periodic cell, of which there are $N_{p} . M_{\kappa}$ is the mass of atom $\kappa$. Substituting this into Eq. 3 we obtain

$$
V_{K S}(R+\delta R)=V_{K S}(R)+\frac{1}{\sqrt{N_{p}}} \sum_{q \nu} G_{q \nu}\left(a_{q \nu}+a_{-q \nu}^{\dagger}\right)
$$

where

$$
G_{q \nu}=\frac{1}{\sqrt{2 \omega_{q \nu}}} \sum_{\kappa} \frac{e_{\kappa \nu}(q)}{\sqrt{M_{\kappa}}} \cdot \sum_{p} e^{i q \cdot R_{p}} \frac{\partial V_{K S}}{\partial R_{\kappa, p}}
$$

This allows us to write down the resulting electronphonon coupling Hamiltonian in second-quantized form 

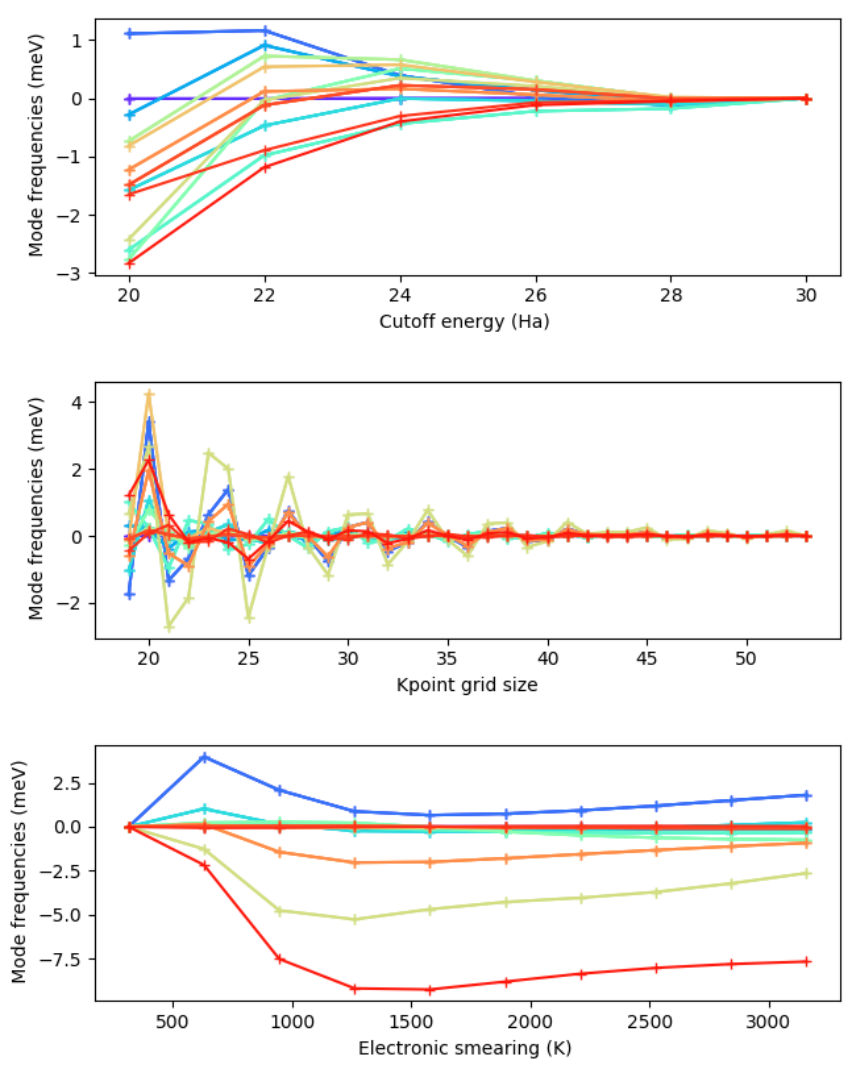

FIG. 19. Convergence behaviour of phonon frequencies for $F m \overline{3} m \mathrm{LaH}_{10}$. Each line corresponds to a different phonon mode.

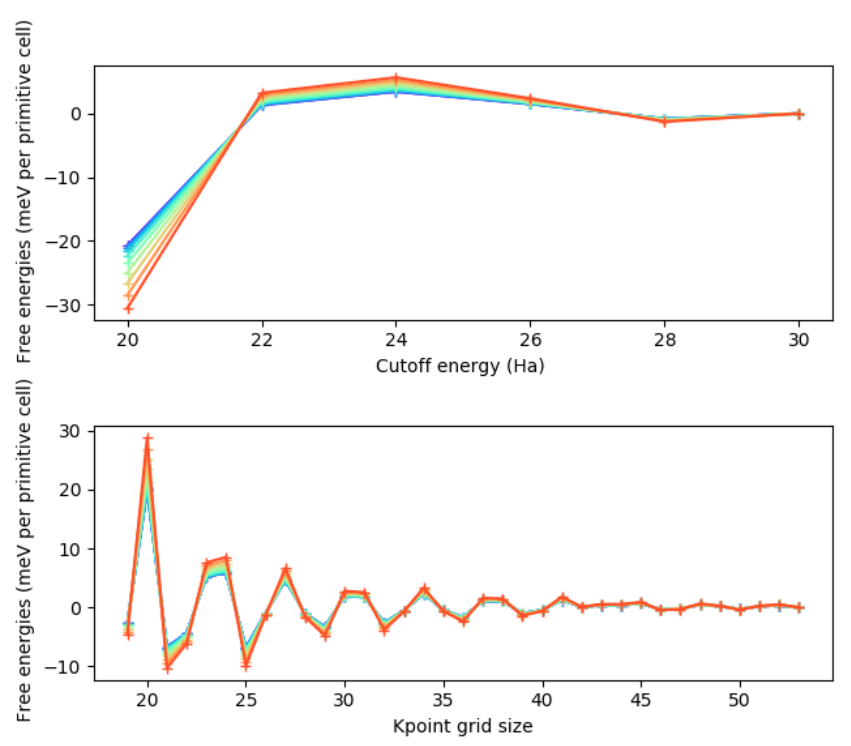

FIG. 20. Convergence behaviour of Helmholtz free energies (including vibrational contributions) for $F m \overline{3} m \mathrm{LaH}_{10}$. Different lines correspond to different temperatures. as

$$
\begin{aligned}
& H_{e p}(\delta R) \\
& =\sum_{n k n^{\prime} k^{\prime}}\left\langle n, k\left|V_{K S}(R+\delta R)-V_{K S}(R)\right| n^{\prime}, k^{\prime}\right\rangle c_{n k}^{\dagger} c_{n^{\prime} k^{\prime}} \\
& =\sum_{q \nu}\left[\sum_{n k n^{\prime} k^{\prime}}\left\langle n, k\left|G_{q \nu}\right| n^{\prime} k^{\prime}\right\rangle c_{n k}^{\dagger} c_{n^{\prime}, k^{\prime}}\right] \frac{a_{q \nu}+a_{-q \nu}^{\dagger}}{\sqrt{N_{p}}}
\end{aligned}
$$

where $c_{n k}^{\dagger}$ creates a Kohn-Sham electron in orbital $n$, wavevector $k$ (i.e., occupies the Bloch state $\left.u_{n k}(x) \exp (i k \cdot x) / \sqrt{N_{p}}\right)$. Substituting our definition of $G_{q \nu}$ we have

$$
\begin{aligned}
& \left\langle n, k\left|G_{q \nu}\right| n^{\prime} k^{\prime}\right\rangle \\
& =\frac{1}{\sqrt{2 \omega_{q \nu}}} \sum_{\kappa} \frac{e_{\kappa \nu}(q)}{\sqrt{M_{\kappa}}} \cdot \sum_{p} e^{i q \cdot R_{p}}\left\langle n, k\left|\frac{\partial V_{K S}}{\partial R_{\kappa, p}}\right| n^{\prime}, k^{\prime}\right\rangle
\end{aligned}
$$

Now

$$
\begin{aligned}
& \left\langle n, k\left|\frac{\partial V_{K S}}{\partial R_{\kappa, p}}\right| n^{\prime}, k^{\prime}\right\rangle \\
& =\frac{1}{N_{p}} \int u_{n k}^{*}(x) e^{-i k \cdot x} \frac{\partial V_{K S}}{\partial R_{\kappa, p}}(x) u_{n^{\prime} k^{\prime}}(x) e^{i k^{\prime} \cdot x} d x \\
& =\frac{1}{N_{p}} \int u_{n k}^{*}\left(x-R_{p}\right) e^{-i k \cdot\left(x-R_{p}\right)} \frac{\partial V_{K S}}{\partial R_{\kappa, p}}\left(x-R_{p}\right) \\
& \quad \times u_{n^{\prime} k^{\prime}}\left(x-R_{p}\right) e^{i k^{\prime} \cdot\left(x-R_{p}\right)} d x \\
& =e^{i R_{p} \cdot\left(k-k^{\prime}\right)} \int_{1^{\text {st }}} u_{n k}^{*}(x) e^{-i k \cdot x} \frac{\partial V_{K S}}{\partial R_{\kappa, 0}}(x) u_{n^{\prime} k^{\prime}}(x) e^{i k^{\prime} \cdot x} d x
\end{aligned}
$$

where in the last line we have used Bloch's theorem and the fact that

$$
\frac{\partial V_{K S}}{\partial R_{\kappa, p}}\left(x-R_{p}\right)=\frac{\partial V_{K S}}{\partial R_{\kappa, 0}}(x)
$$

where $R_{\kappa, 0}$ is the position of atom $\kappa$ in the first unit cell. We may now write Eq. 8 as

$$
\begin{aligned}
& \left\langle n, k\left|G_{q \nu}\right| n^{\prime} k^{\prime}\right\rangle \\
& =\frac{1}{\sqrt{2 \omega_{q \nu}}} \sum_{\kappa} \frac{e_{\kappa \nu}(q)}{\sqrt{M_{\kappa}}} \cdot\left\langle n, k\left|\frac{\partial V_{K S}}{\partial R_{\kappa, 0}}\right| n^{\prime}, k^{\prime}\right\rangle_{\mathrm{uc}} \\
& \times \underbrace{\sum_{p} e^{i\left(q+\left(k-k^{\prime}\right)\right) \cdot R_{p}}}_{N_{p} \delta_{q, k-k^{\prime}}}
\end{aligned}
$$

where the subscript "uc" on the ket means integration only over the first unit cell. Finally we obtain the DFT electron-phonon coupling Hamiltonian

$$
\begin{array}{r}
H_{e p}=\frac{1}{\sqrt{N_{p}}} \sum_{q \nu k n m}\left\langle m, k+q\left|G_{q \nu, \mathrm{uc}}\right| n, k\right\rangle_{\mathrm{uc}} \\
\times c_{m, k+q}^{\dagger} c_{n, k}\left(a_{q \nu}+a_{-q \nu}^{\dagger}\right)
\end{array}
$$

where we have defined

$$
G_{q \nu, \mathrm{uc}}=\frac{1}{\sqrt{2 \omega_{q \nu}}} \sum_{\kappa} \frac{e_{\kappa \nu}(q)}{\sqrt{M_{\kappa}}} \cdot \frac{\partial V_{K S}}{\partial R_{\kappa, 0}}
$$




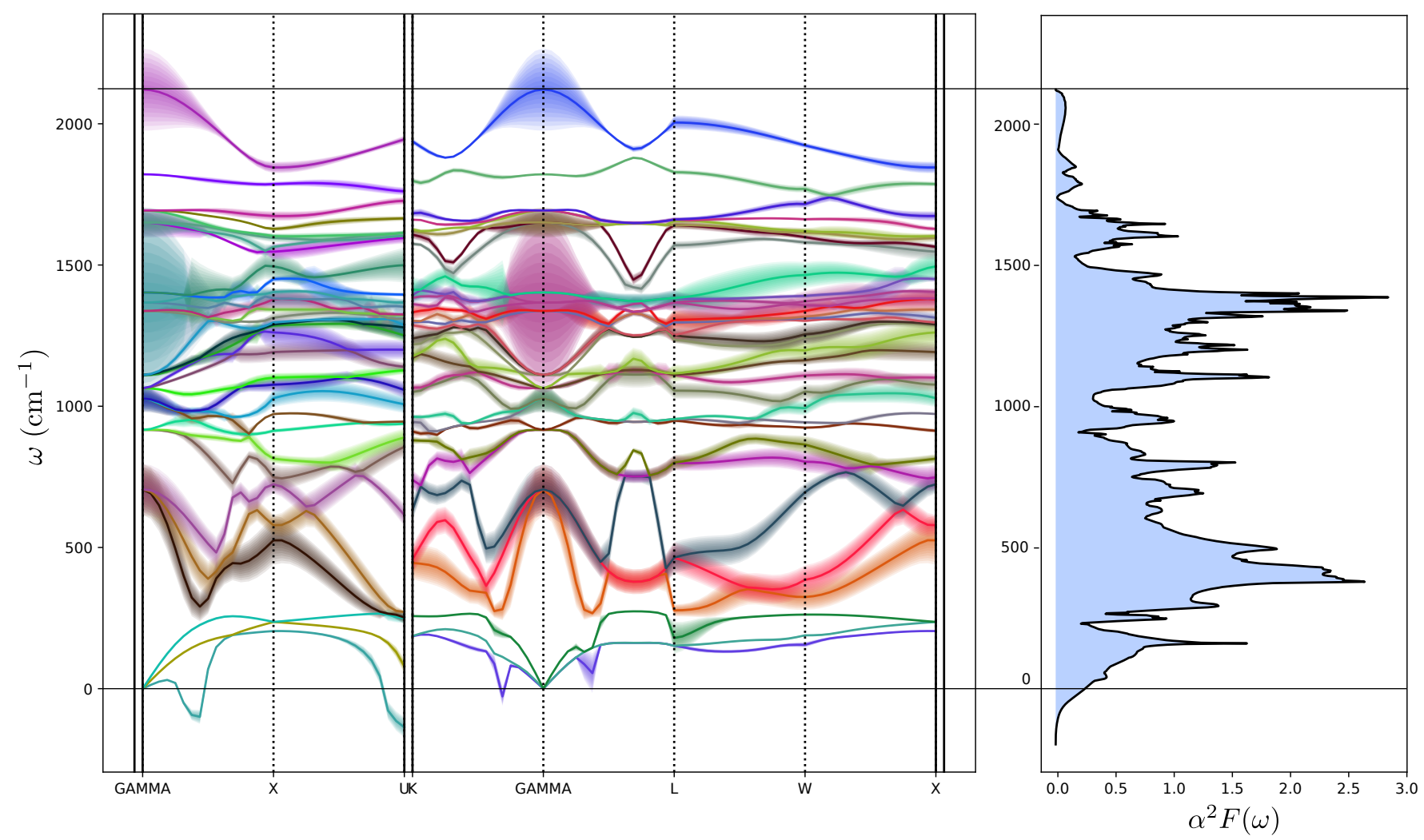

FIG. 21. The phonon dispersion, showing linewidth broadening and the Eliashberg function for the $F m \overline{3} m$ phase of LaH $\mathrm{H}_{10}$ at $200 \mathrm{GPa}$.
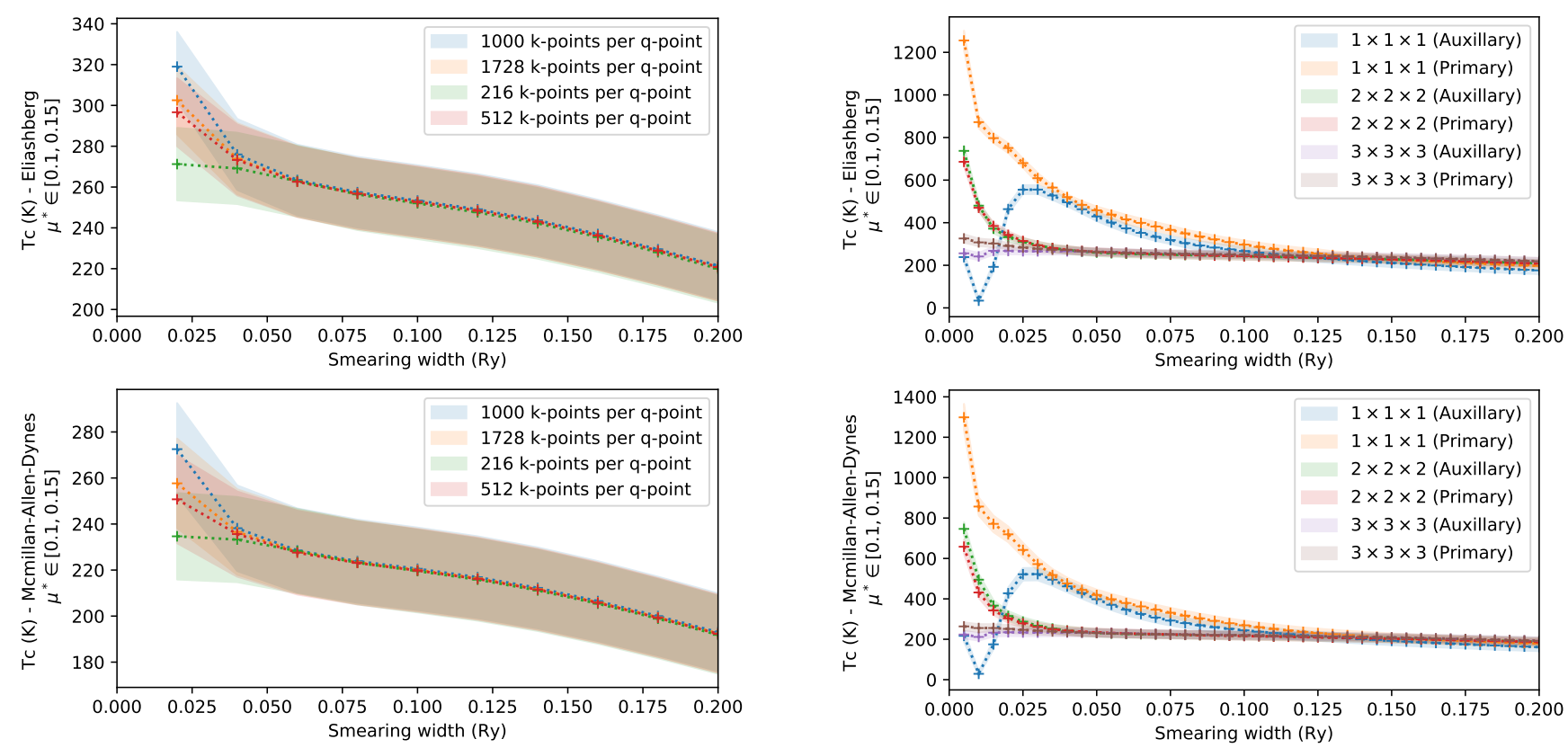

FIG. 22. $T_{C}$ vs. double-delta smearing for $F m \overline{3} m \mathrm{YH}_{10}$, calculated using different sized k-point grids with a fixed $3 \times$ $3 \times 3$ q-point grid.

FIG. 23. $T_{C}$ vs. double-delta smearing for $F m \overline{3} m \mathrm{YH}_{10}$, calculated using different sized q-point grids. The results for primary/auxiliary k-point grids for each q-point grid are shown (primary $=512 \mathrm{k}$-points per q-point, auxiliary $=261 \mathrm{k}$-points per q-point). 

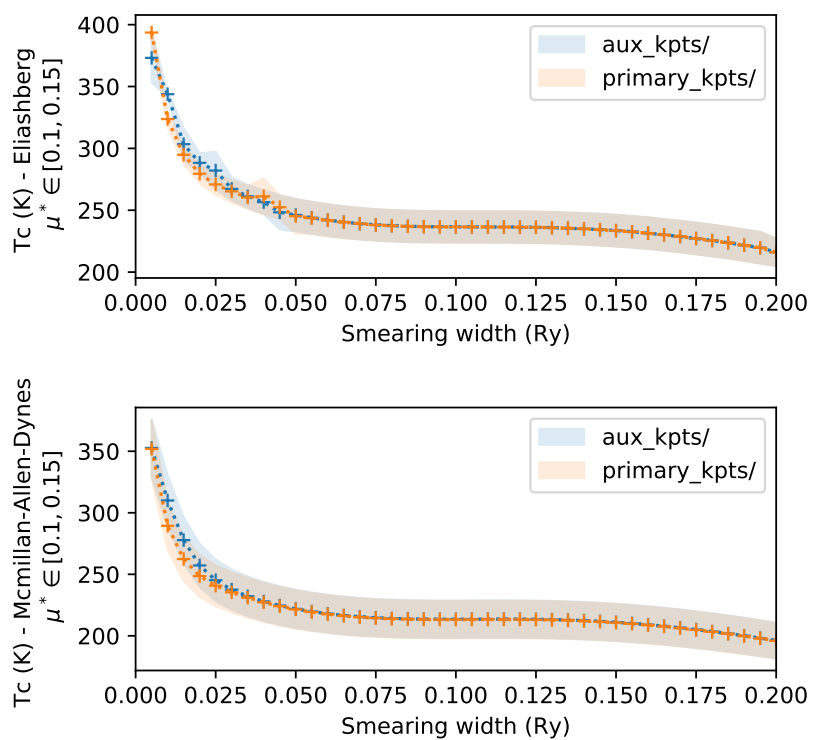

FIG. 24. $T_{C}$ vs. double-delta smearing for $F m \overline{3} m \mathrm{LaH}_{10}$, calculated using different sized k-point grids with a fixed $3 \times$ $3 \times 3$ q-point grid.

This allows us to write down the Hamiltonian for an interacting Kohn-Sham-electron-phonon system, correct to first order in electron-phonon coupling constants $g_{m n \nu}(k, q)=\left\langle m, k+q\left|G_{q \nu, \mathrm{uc}}\right| n, k\right\rangle_{\mathrm{uc}}$ :

$$
\begin{gathered}
H=\underbrace{\sum_{k n} \epsilon_{n k} c_{n k}^{\dagger} c_{n k}}_{\text {Electronic dispersion }}+\underbrace{\sum_{q \nu} \omega_{q \nu}\left(a_{q \nu}^{\dagger} a_{q \nu}+\frac{1}{2}\right)}_{\text {phonon dispersion }}+ \\
\underbrace{\frac{1}{\sqrt{N_{p}}} \sum_{k q m n \nu} g_{m n \nu}(k, q) c_{m, k+q}^{\dagger} c_{n k}\left(a_{q \nu}+a_{-q \nu}^{\dagger}\right)}_{\text {electron-phonon coupling }} .
\end{gathered}
$$

From the parameters in this Hamiltonian we can also define the electron-phonon coupling strength associated with each phonon mode, $\lambda_{q \nu}$, and the isotropic Eliashberg spectral function, $\alpha^{2} F(\omega)$

$$
\begin{aligned}
& \lambda_{q, \nu}=\frac{1}{N\left(\epsilon_{F}\right) \omega_{q \nu} \Omega_{\mathrm{BZ}}} \\
& \times \sum_{n m} \int_{\mathrm{BZ}}\left|g_{m n \nu}(k, q)\right|^{2} \delta\left(\epsilon_{n, k}-\epsilon_{F}\right) \delta\left(\epsilon_{m, k+q}-\epsilon_{F}\right) d k \\
& \alpha^{2} F(\omega)=\frac{1}{2 \Omega_{\mathrm{BZ}}} \sum_{\nu} \int_{\mathrm{BZ}} \omega_{q \nu} \lambda_{q \nu} \delta\left(\omega-\omega_{q \nu}\right) d q
\end{aligned}
$$

from which we may calculate the critical temperature by solution of the Eliashberg equations [37. The only additional requirement is the Morel-Anderson pseudopotential [49, which we treat as an empirical parameter with values between 0.1 and 0.15 . 
[1] N. W. Ashcroft, Phys. Rev. Lett. 21, 1748 (1968).

[2] J. M. McMahon, M. A. Morales, C. Pierleoni, and D. M. Ceperley, Rev. Mod. Phys 84, 1607 (2012).

[3] H.-K. Mao and R. J. Hemley, Science 244, 1462 (1989).

[4] M. Eremets and I. Troyan, Nature Materials 10, 927 (2011).

[5] P. Dalladay-Simpson, R. T. Howie, and E. Gregoryanz, Nature 529, 63 (2016).

[6] R. P. Dias and I. F. Silvera, Science 355, 715 (2017).

[7] P. Loubeyre, F. Occelli, and P. Dumas, Nature 577, 631 (2020).

[8] N. W. Ashcroft, Phys. Rev. Lett. 92, 187002 (2004).

[9] D. Duan, Y. Liu, Y. Ma, Z. Shao, B. Liu, and T. Cui, Natl. Sci. Rev 4, 121 (2017).

[10] E. Zurek and T. Bi, J. Chem. Phys. 150, 050901 (2019).

[11] J. A. Flores-Livas, L. Boeri, A. Sanna, G. Profeta, R. Arita, and M. Eremets, Physics Reports (2020), https://doi.org/10.1016/j.physrep.2020.02.003.

[12] L. Boeri and G. B. Bachelet, J. Phys: Condens. Matt. 31, 234002 (2019).

[13] A. R. Oganov, C. J. Pickard, Q. Zhu, and R. J. Needs, Nature Reviews Materials 4, 331 (2019).

[14] R. J. Needs and C. J. Pickard, APL Materials 4, 053210 (2016).

[15] C. J. Pickard, I. Errea, and M. I. Eremets, Annual Review of Condensed Matter Physics 11, 57 (2020).

[16] A. P. Durajski and R. Szczesniak, Supercond. Sci. and Tech. 27, 115012 (2014).

[17] D. Y. Kim, R. H. Scheicher, and R. Ahuja, Phys. Rev. Lett. 103, 077002 (2009).

[18] Y. Li, J. Hao, H. Liu, J. S. Tse, Y. Wang, and Y. Ma, Scientific Reports 5, 9948 (2015).

[19] C. Heil, S. di Cataldo, G. B. Bachelet, and L. Boeri, Phys. Rev. B 99, 220502(R) (2019).

[20] H. Liu, I. I. Naumov, R. Hoffmann, N. W. Ashcroft, and R. J. Hemley, Proc. Nat. Acad. Sci. 114, 6990 (2017).

[21] F. Peng, Y. Sun, C. J. Pickard, R. J. Needs, Q. Wu, and Y. Ma, Phys. Rev. Lett. 119, 107001 (2017).

[22] Z. M. Geballe, H. Liu, A. K. Mishra, M. Ahart, M. Somayazulu, Y. Meng, M. Baldini, and R. J. Hemley, Angewandte Chemie International Edition 57, 688 (2018).

[23] H. Liu, I. I. Naumov, Z. M. Geballe, M. Somayazulu, J. S. Tse, and R. J. Hemley, Phys. Rev. B 98, 100102(R) (2018).

[24] I. Errea, F. Belli, L. Monacelli, A. Sanna, T. Koretsune, T. Tadano, R. Bianco, M. Calandra, R. Arita, F. Mauri, and J. A. Flores-Livas, Nature 578, 66 (2020).

[25] M. Somayazulu, M. Ahart, A. K. Mishra, Z. M. Geballe, M. Baldini, Y. Meng, V. V. Struzhkin, and R. J. Hemley, Phys. Rev. Lett. 122, 027001 (2019).

[26] A. P. Drozdov, P. P. Kong, V. S. Minkov, S. P. Besedin, M. A. Kuzovnikov, S. Mozaffari, L. Balicas, F. F. Balakirev, D. E. Graf, V. B. Prakapenka, E. Greenberg, D. A. Knyazev, M. Tkacz, and M. I. Eremets, Nature 569, 528 (2019).

[27] N. P. Salke, M. M. D. Esfahani, Y. Zhang, I. A. Kruglov, J. Zhou, Y. Wang, E. Greenberg, V. B. Prakapenka, J. Liu, A. R. Oganov, and J.-F. Lin, Nature Communications 10, 1 (2019).

[28] X. Li, X. Huang, D. Duan, C. J. Pickard, D. Zhou, H. Xie, Q. Zhuang, Y. Huang, Q. Zhou, B. Liu, and T. Cui,
Nature Communications 10, 3461 (2019).

[29] D. Zhou, D. V. Semenok, D. Duan, H. Xie, W. Chen, X. Huang, X. Li, B. Liu, A. R. Oganov, and T. Cui, Science Advances 6 (2020), 10.1126/sciadv.aax6849, https://advances.sciencemag.org/content/6/9/eaax6849.full.pdf

[30] D. Zhou, D. V. Semenok, H. Xie, X. Huang, D. Duan, A. Aperis, P. M. Oppeneer, M. Galasso, A. I. Kartsev, A. G. Kvashnin, A. R. Oganov, and T. Cui, Journal of the American Chemical Society 142, 2803 (2020).

[31] P. Hohenberg and W. Kohn, Phys. Rev 136, B864 (1964).

[32] W. Kohn and L. J. Sham, Phys. Rev 140, A1133 (1965).

[33] F. Giustino, Rev. Mod. Phys. 89, 015003 (2017).

[34] P. Giannozzi, S. Baroni, N. Bonini, M. Calandra, R. Car, C. Cavazzoni, D. Ceresoli, G. L. Chiarotti, M. Cococcioni, I. Dabo, A. Dal Corso, S. de Gironcoli, S. Fabris, G. Fratesi, R. Gebauer, U. Gerstmann, C. Gougoussis, A. Kokalj, M. Lazzeri, L. Martin-Samos, N. Marzari, F. Mauri, R. Mazzarello, S. Paolini, A. Pasquarello, L. Paulatto, C. Sbraccia, S. Scandolo, G. Sclauzero, A. P. Seitsonen, A. Smogunov, P. Umari, and R. M. Wentzcovitch, Journal of Physics: Condensed Matter 21, 395502 (19pp) (2009)

[35] P. Giannozzi, O. Andreussi, T. Brumme, O. Bunau, M. B. Nardelli, M. Calandra, R. Car, C. Cavazzoni, D. Ceresoli, M. Cococcioni, N. Colonna, I. Carnimeo, A. D. Corso, S. de Gironcoli, P. Delugas, R. A. DiStasio, A. Ferretti, A. Floris, G. Fratesi, G. Fugallo, R. Gebauer, U. Gerstmann, F. Giustino, T. Gorni, J. Jia, M. Kawamura, H.-Y. Ko, A. Kokalj, E. Küçükbenli, M. Lazzeri, M. Marsili, N. Marzari, F. Mauri, N. L. Nguyen, H.V. Nguyen, A. O. de-la Roza, L. Paulatto, S. Poncé, D. Rocca, R. Sabatini, B. Santra, M. Schlipf, A. P. Seitsonen, A. Smogunov, I. Timrov, T. Thonhauser, P. Umari, N. Vast, X. Wu, and S. Baroni, Journal of Physics: Condensed Matter 29, 465901 (2017).

[36] Our optimisations have been submitted to the QUANTUM ESPRESSO project.

[37] G. M. Eliashberg, Sov. Phys. JETP 11, 696 (1960).

[38] http://elk.sourceforge.net/, the ELK FP-LAPW code.

[39] M. Wierzbowska, S. de Gironcoli, and P. Giannozzi, arXiv:cond-mat/0504077 (2005).

[40] J. P. Perdew, K. Burke, and M. Ernzerhof, Phys. Rev. Lett. 77, 3865 (1996).

[41] P. Blaha, K. Schwarz, G. K. Madsen, D. Kvasnicka, and J. Luitz, http://www.wien2k.at/reg_user/textbooks/ usersguide.pdf/ (2001), wien2k user guide.

[42] See supplementary information.

[43] I. A. Troyan, D. V. Semenok, A. G. Kvashnin, A. V. Sadakov, O. A. Sobolevskiy, V. M. Pudalov, A. G. Ivanova, V. B. Prakapenka, E. Greenberg, A. G.Gavriliuk, V. V. Struzhkin, A. Bergara, I. Errea, R. Bianco, M. Calandra, F. Mauri, L. Monacelli, R. Akashi, and A. R. Oganov, arXiv preprint arXiv:1908.01534 (2020).

[44] P. P. Kong, V. S. Minkov, M. A. Kuzovnikov, S. P. Besedin, A. P. Drozdov, S. Mozaffari, L. Balicas, F. F. Balakirev, V. B. Prakapenka, E. Greenberg, D. A. Knyazev, and M. I. Eremets, arXiv preprint arXiv:1909.10482 (2019).

[45] C. J. Pickard and R. J. Needs, J. Phys: Condens. Matt. 23, 053201 (2011). 
[46] S. J. Clark, M. D. Segall, C. J. Pickard, P. J. Hasnip, M. I. J. Probert, K. Refson, and M. C. Payne, Zeitschrift für Kristallographie-Crystalline Materials 220, 567 (2005).

[47] M. Rutter, Computer Physics Communications 225, 174 (2018)

[48] F. Birch, Phys. Rev. 71, 809 (1947).
[49] P. Morel and P. W. Anderson, Phys. Rev. 125, 1263 (1962)

[50] I. A. Kruglov, D. V. Semenok, H. Song, R. L. Szczesniak, I. A. Wrona, R. Akashi, M. M. D. Esfahani, D. Duan, T. Cui, A. G. Kvashnin, and A. R. Oganov, Phys. Rev. B 101, 024508 (2020).

[51] C. B. Barber, D. P. Dobkin, and H. T. Huhdanpaa, ACM Trans. on Mathematical Software 22, 469 (1996).

[52] C. Heil, G. B. Bachelet, and L. Boeri, Phys. Rev. B 97, 214510 (2018). 